\title{
Vector SIMP dark matter with approximate custodial symmetry
}

\author{
Soo-Min Choi, ${ }^{a}$ Hyun Min Lee, ${ }^{a, b}$ Yann Mambrini ${ }^{c}$ and Mathias Pierre ${ }^{d, e}$ \\ ${ }^{a}$ Department of Physics, Chung-Ang University, \\ Seoul 06974, Korea \\ ${ }^{b}$ School of Physics, Korea Institute for Advanced Study, \\ Seoul 02455, Korea \\ ${ }^{c}$ Laboratoire de Physique Théorique (UMR8627), CNRS, \\ Univ. Paris-Sud, Université Paris-Saclay, \\ 91405 Orsay, France \\ ${ }^{d}$ Instituto de Física Teórica (IFT) UAM-CSIC, \\ Campus de Cantoblanco, 28049 Madrid, Spain \\ ${ }^{e}$ Departamento de Física Teórica, Universidad Autónoma de Madrid (UAM), \\ Campus de Cantoblanco, 28049 Madrid, Spain \\ E-mail: soominchoi90@gmail.com, hminlee@cau.ac.kr, \\ yann.mambrini@th.u-psud.fr, mathias.pierre@uam.es
}

Abstract: We consider a novel scenario for Vector Strongly Interacting Massive Particle (VSIMP) dark matter with local $\mathrm{SU}(2)_{X} \times \mathrm{U}(1)_{Z^{\prime}}$ symmetry in the dark sector. Similarly to the Standard Model (SM), after the dark symmetry is broken spontaneously by the VEVs of dark Higgs fields, the approximate custodial symmetry determines comparable but split masses for $\mathrm{SU}(2)_{X}$ gauge bosons. In this model, we show that the $\mathrm{U}(1)_{Z^{\prime}}$-charged gauge boson of $\mathrm{SU}(2)_{X}\left(X_{ \pm}\right)$becomes a natural candidate for SIMP dark matter, annihilating through $3 \rightarrow 2$ or forbidden $2 \rightarrow 2$ annihilations due to gauge self-interactions. On the other hand, the $\mathrm{U}(1)_{Z^{\prime}}$-neutral gauge boson of $\mathrm{SU}(2)_{X}$ achieves the kinetic equilibrium of dark matter through a gauge kinetic mixing between $\mathrm{U}(1)_{Z^{\prime}}$ and SM hypercharge. We present the parameter space for the correct relic density in our model and discuss in detail the current constraints and projections from colliders and direct detection experiments.

Keywords: Beyond Standard Model, Cosmology of Theories beyond the SM, Gauge Symmetry

ArXiv EPrint: 1904.04109 


\section{Contents}

1 Introduction 1

2 Model 2

3 Dark gauge boson masses 4

3.1 Dark custodial symmetry and its breaking 4

$\begin{array}{lll}3.2 & \text { Split dark gauge bosons } & 6\end{array}$

$\begin{array}{lll}3.3 & \text { Degenerate dark gauge bosons } & 7\end{array}$

4 Dark matter annihilations with self-interactions $\quad 8$

4.1 Boltzmann equations 8

$4.2 \quad 3 \rightarrow 2$ annihilations 8

$\begin{array}{lll}4.3 & \text { Forbidden } 2 \rightarrow 2 \text { annihilations } & 11\end{array}$

4.4 DM self-scattering 13

$5 \quad Z^{\prime}$ portal couplings for dark matter $\quad 14$

$\begin{array}{lll}5.1 & \text { General current interactions with } Z^{\prime} \text { portal } & 14\end{array}$

$\begin{array}{lll}5.2 & \text { Kinetic equilibrium } & 17\end{array}$

$\begin{array}{lll}5.3 & \text { SM } 2 \rightarrow 2 \text { annihilations and direct detection } & 19\end{array}$

6 Conclusions 20

$\begin{array}{ll}\text { A General dark gauge boson masses } & 20\end{array}$

B Dark Higgs masses $\quad 22$

\section{Introduction}

Despite indirect evidences for the presence of dark matter in our Universe [1, 2], the nature of dark matter is still elusive. The absence of signals in direct detection experiments like LUX [3], PANDAX [4] or more recently XENON1T [5] questions the WIMP (Weakly Interacting Massive Particle) paradigm. The simplest extensions, involving minimal ingredients as Higgs-portal [6-19], $Z$-portal [20-23] or even $Z^{\prime}$-portal [24-27], etc, are already excluded for Beyond the Standard Model (BSM) scale below $\sim 3 \mathrm{TeV}$, once we combine cosmological, accelerators and direct detection constraints (see [28] for a recent review on the subject and [29] for the recent global fits for Higgs-portal dark matter). In this context, one needs to develop extensions of the Standard Model beyond the WIMP paradigm. 
One possibility for going beyond the WIMP paradigm is to modify drastically the thermal history of dark matter, allowing for extremely feeble couplings between the visible and dark sectors. The correct relic abundance is then ensured through the freeze-in mechanism [30-45]. Another possibility proposed recently is to allow for large self-interacting couplings generating $3 \rightarrow 2$ or $2 \rightarrow 2$ forbidden channels [46-61], which can then provide solutions to some of small-scale problems at galaxy scales [62-68]. These Strongly Interacting Massive Particle (SIMP) scenarios, naturally present in the case of non-abelian dark matter, open up a complete new range of parameter space that still needs to be explored.

We propose in this work to analyse in detail a simple $\mathrm{SU}(2)_{X} \times \mathrm{U}(1)_{Z^{\prime}}$ extension of the Standard Model (SM), where the charged non-abelian vector boson $X_{ \pm}$is the dark matter candidate, while the $Z_{\mu}^{\prime}$ boson plays a role of the portal between the visible and hidden sectors through its kinetic mixing with the SM hypercharge gauge boson $B_{\mu}$. Our construction provides a novel and efficient mechanism for maintaining VSIMP dark matter in kinetic equilibrium during freeze-out, being consistent with the observed relic density. We will discuss the interplay between correct relic density and kinetic equilibrium in constraining the parameter space, which can be tested at current and future experiments.

Our paper is organized as follows. We describe the model in the first section and compute its spectrum in section 3. Section 4 is devoted to the computation of dark matter abundance through the $3 \rightarrow 2$ and $2 \rightarrow 2$ forbidden channels while a combined analysis including constraints from direct detection and accelerators searches is presented in section 5 . We then conclude. There are two appendices dealing with general masses for gauge and Higgs bosons in the dark sector.

\section{$2 \quad$ Model}

We consider models with a local $\mathrm{SU}(2)_{X} \times \mathrm{U}(1)_{Z^{\prime}}$ symmetry in the dark sector, "dark" in the sense that the Standard Model particles are not charged under these transformations. The dark Higgs sector is composed of a singlet scalar $S$ and a nontrivial representation scalar $H_{X}$ under $\mathrm{SU}(2)_{X}$, with $\mathrm{U}(1)_{Z^{\prime}}$ charges given by $q_{S}$ and $q_{H_{X}}$, respectively. Without loss of generality, we choose $q_{H_{X}}=I_{3}$ where $I_{3}$ is the dark isospin of $H_{X}$.

The Lagrangian for the dark sector in our model is then given by

$$
\mathcal{L}=-\frac{1}{4} \vec{X}_{\mu \nu} \cdot \vec{X}^{\mu \nu}-\frac{1}{4} Z_{\mu \nu}^{\prime} Z^{\prime \mu \nu}+\mathcal{L}_{\text {scalar }}+\mathcal{L}_{Z^{\prime}-\text { portal }}+\mathcal{L}_{H-\text { portal }}
$$

where the field strength tensors are $\vec{X}_{\mu \nu}=\partial_{\mu} \vec{X}_{\nu}-\partial_{\nu} \vec{X}_{\mu}+g_{X}\left(\vec{X}_{\mu} \times \vec{X}_{\nu}\right)$ and $Z_{\mu \nu}^{\prime}=$ $\partial_{\mu} Z_{\nu}^{\prime}-\partial_{\nu} Z_{\mu}^{\prime}$. The scalar part of the Lagrangian containing the SM Higgs doublet $H$ is given by

$$
\begin{aligned}
\mathcal{L}_{\text {scalar }}= & \left|D_{\mu} S\right|^{2}+m_{S}^{2}|S|^{2}-\lambda_{S}|S|^{4}+\left|D_{\mu} H_{X}\right|^{2} \\
& +m_{H_{X}}^{2}\left|H_{X}\right|^{2}-\lambda_{H_{X}}\left|H_{X}\right|^{4}+\tilde{\lambda}_{H_{X}}\left(H_{X}^{\dagger} t_{i} H_{X}\right)\left(H_{X}^{\dagger} t_{i} H_{X}\right)+V_{\text {mix }}\left(S, H_{X}\right) \\
& +\left|D_{\mu} H\right|^{2}+m_{H}^{2}|H|^{2}-\lambda_{H}|H|^{4}
\end{aligned}
$$

where the covariant derivatives for $S$ and $H_{X}$ are

$$
D_{\mu}=\partial_{\mu}-i g_{X} \vec{t} \cdot \vec{X}_{\mu}-i g_{Z^{\prime}} q_{Z^{\prime}} Z_{\mu}^{\prime}
$$


with $t^{i}(i=1,2,3)$ being $\mathrm{SU}(2)_{X}$ generators satisfying $\left[t^{i}, t^{j}\right]=i \epsilon^{i j k} t^{k}, V_{\text {mix }}$ the scalar potential including mixing quartic couplings between dark Higgs fields, ${ }^{1}$ and $q_{Z^{\prime}}=q_{S}$ or $q_{H_{X}}$. We also introduce a gauge kinetic mixing between $Z^{\prime}$ and hypercharge gauge bosons,

$$
\mathcal{L}_{Z^{\prime} \text {-portal }}=-\frac{1}{2} \sin \xi Z_{\mu \nu}^{\prime} B^{\mu \nu}
$$

This mixing will play the role of the portal between the dark sector and the Standard Model for the thermalization process. For completeness, we allow for Higgs portal couplings between the dark scalars and the SM Higgs,

$$
\mathcal{L}_{H \text {-portal }}=-\lambda_{S H}|S|^{2}|H|^{2}-\lambda_{H_{X} H}\left|H_{X}\right|^{2}|H|^{2} .
$$

Moreover, developing eq. (2.1), we extract self-interacting interactions of dark gauge bosons [61],

$$
\begin{aligned}
\mathcal{L}_{\text {self }}= & -\frac{1}{2} g_{X}\left(\partial_{\mu} \vec{X}_{\nu}-\partial_{\nu} \vec{X}_{\mu}\right) \cdot\left(\vec{X}^{\mu} \times \vec{X}^{\nu}\right)-\frac{1}{4} g_{X}^{2}\left(\vec{X}_{\mu} \cdot \vec{X}^{\mu}\right)^{2} \\
& +\frac{1}{4} g_{X}^{2}\left(\vec{X}_{\nu} \cdot \vec{X}^{\mu}\right)\left(\vec{X}_{\mu} \cdot \vec{X}^{\nu}\right) \\
\equiv & \mathcal{L}_{3}+\mathcal{L}_{4}
\end{aligned}
$$

with

$$
\begin{aligned}
\mathcal{L}_{3}= & -i g_{X}\left[\left(\partial^{\mu} X^{\nu}-\partial^{\nu} X^{\mu}\right) X_{\mu}^{\dagger} X_{3, \nu}-\left(\partial^{\mu} X^{\nu \dagger}-\partial^{\nu} X^{\mu \dagger}\right) X_{\mu} X_{3, \nu}\right. \\
& \left.+X_{\mu} X_{\nu}^{\dagger}\left(\partial^{\mu} X_{3}^{\nu}-\partial^{\nu} X_{3}^{\mu}\right)\right] \\
\mathcal{L}_{4}= & -\frac{g_{X}^{2}}{2}\left[\left(X_{\mu}^{\dagger} X^{\mu}\right)^{2}-X_{\mu}^{\dagger} X^{\mu \dagger} X_{\nu} X^{\nu}\right]-g_{X}^{2}\left(X_{\mu}^{\dagger} X^{\mu} X_{\nu, 3} X_{3}^{\nu}-X_{\mu}^{\dagger} X_{3}^{\mu} X_{\nu} X_{3}^{\nu}\right)
\end{aligned}
$$

where $X_{\mu} \equiv\left(X_{1, \mu}+i X_{2, \mu}\right) / \sqrt{2}$ and its complex conjugate, $X_{\mu}^{\dagger} \equiv\left(X_{1, \mu}-i X_{2, \mu}\right) / \sqrt{2}$. See also refs. $[69,70]$ for some of non-abelian dark matter candidates.

After $\mathrm{SU}(2)_{X} \times \mathrm{U}(1)_{Z^{\prime}}$ is broken by the VEVs of dark scalars, the dark gauge bosons have nonzero masses, and $X_{1,2, \mu}$ gauge bosons are combined to be a complex gauge boson $X_{\mu}$ with nonzero charge under $\mathrm{U}(1)_{Z^{\prime}}$. Thus, $X_{1,2, \mu}$ gauge bosons, if the lightest particle with $\mathrm{U}(1)_{Z^{\prime}}$ charge in the dark sector, can be a dark matter candidate. Indeed, as the Standard Model is neutral under $\mathrm{U}(1)_{Z^{\prime}}$, no decay modes of $X_{\mu}$ into the visible sector are allowed due to the DM $Z_{2}$ symmetry after spontaneous breaking of $\mathrm{U}(1)_{Z^{\prime}}$. On the other hand, as it happens in the Standard Model with $W^{ \pm}$and $Z^{0}, X_{3 \mu}$ has a different mass from $X_{1,2 \mu}$ due to the VEV of $H_{X}$ charged under the $\mathrm{U}(1)_{Z^{\prime}}$, and it can couple to the SM particles through the dark Weinberg angle in combination with the gauge kinetic mixing, as it will be discussed later. Our situation is a mirror case of the Standard Model, except for the presence of an extra-singlet $S$ avoiding the presence of a massless gauge boson.

\footnotetext{
${ }^{1}$ Notice that we can set $\tilde{\lambda}_{H_{X}}=0$ when $H_{X}$ is a doublet.
} 


\section{Dark gauge boson masses}

In this section, we discuss the approximate custodial symmetry in the $\mathrm{SU}(2)_{X}$ gauge sector with general dark Higgs representations and show the effect of the $Z^{\prime}$ gauge boson on the mass splitting between dark-neutral and charged gauge bosons.

\subsection{Dark custodial symmetry and its breaking}

We considered the expansions of dark Higgs fields about nonzero VEVs by a singlet $S=\frac{1}{\sqrt{2}}\left(v_{S}+s\right)$, and a Higgs field $H_{X}$ in several representation of $\mathrm{SU}(2)_{X}$ : a doublet $\Phi=\left(0, \frac{1}{\sqrt{2}}\left(v_{\Phi}+h_{\Phi}\right)\right)^{T}$, a triplet $T=\left(h^{++}, 0, \frac{1}{\sqrt{2}}\left(v_{T}+h_{T}\right)\right)^{T}$ or a quadruplet $Q_{4}=$ $\left(h^{(3)}, h^{(2)}, 0, \frac{1}{\sqrt{2}}\left(v_{Q_{4}}+h_{Q_{4}}\right)\right)^{T}$ or a quintuplet $Q_{5}=\left(h^{(4)}, h^{(3)}, h^{(2)}, 0, \frac{1}{\sqrt{2}}\left(v_{Q_{5}}+h_{Q_{5}}\right)\right)^{T}$, in unitary gauge. We present general masses for dark gauge bosons in appendix $\mathrm{A}$, while the expression for the dark Higgs masses and the mixing between the dark Higgs and the SM Higgs are given in appendix B.

First, due to the VEV of the dark Higgs $H_{X}$ in a nontrivial representation of $\mathrm{SU}(2)_{X}$, such as $\Phi, T, Q_{4}$ or $Q_{5}$, masses of dark-charged gauge bosons, $X_{\mu}, X_{\mu}^{\dagger}$, are given by

$$
m_{X}^{2}=\frac{1}{2} g_{X}^{2} I v_{I}^{2}
$$

with $I=\frac{1}{2}, 1, \frac{3}{2}, 2$ respectively. For a vanishing $Z^{\prime}$ charge of the dark Higgs $H_{X}$ or in the limit of a vanishing $g_{Z^{\prime}}$, the dark-neutral gauge boson has mass

$$
m_{X_{3}, 0}^{2}=g_{X}^{2} I^{2} v_{I}^{2}
$$

leading to the mass relations due to dark custodial symmetry,

$$
\frac{m_{X}^{2}}{m_{X_{3}, 0}^{2}}=\frac{1}{2 I} .
$$

Notice that we recover the $M_{Z}=M_{W}$ relation of the Standard Model with a Higgs doublet $\left(I=\frac{1}{2}\right)$ in the case of a null Weinberg angle. As a result, if $I>\frac{1}{2}$, the dark-charged gauge boson $X$ can be the lightest gauge boson in the dark sector, becoming a candidate for non-abelian dark matter. On the other hand, once taking into consideration the VEV of $S, Z^{\prime}$ gauge boson can decouple from the $X$ spectrum due to the contribution of $v_{S}$ to its mass. To maintain the thermal equilibrium of $X_{\mu}$ with the Standard Model bath, one needs to consider first its coupling to $Z^{\prime}$.

Indeed, in order to communicate between non-abelian dark matter and SM by renormalizable couplings, it is sufficient to consider the dark Higgs $H_{X}$ with nonzero $Z^{\prime}$ charge and take a sizable $g_{Z^{\prime}}$. Then, the mass matrix for neutral gauge bosons in the basis $\left(Z_{\mu}^{\prime}, X_{3 \mu}\right)$, receives a correction term violating the dark custodial symmetry, as follows,

$$
M_{2 \times 2}^{2}=m_{X_{3}}^{2}\left(\begin{array}{cc}
\alpha s_{X}^{2} & -s_{X} c_{X} \\
-s_{X} c_{X} & c_{X}^{2}
\end{array}\right)
$$


where $m_{X_{3}}^{2} \equiv\left(g_{X}^{2}+g_{Z^{\prime}}^{2}\right) I^{2} v_{I}^{2}=m_{X_{3}, 0}^{2}+g_{Z^{\prime}}^{2} I^{2} v_{I}^{2}, c_{X} \equiv \cos \theta_{X}$ and $s_{X} \equiv \sin \theta_{X}$, with $\sin \theta_{X}=g_{Z^{\prime}} / \sqrt{g_{X}^{2}+g_{Z^{\prime}}^{2}}$, and

$$
\alpha \equiv 1+\frac{q_{S}^{2} v_{S}^{2}}{I^{2} v_{I}^{2}} .
$$

Unlike in the SM, there exists a singlet scalar $S$ contributing to the $Z^{\prime}$ mass. The most general dark gauge boson masses with VEVs of Higgs fields in arbitrary representations are given in the appendix A.

In the absence of the gauge kinetic mixing, the mass matrix (3.4) can be diagonalized explicitly by introducing a dark Weinberg angle as in the SM. Performing a rotation of dark gauge fields to mass eigenstates, ${ }^{2} \tilde{Z}_{\mu}^{\prime}, \tilde{X}_{3 \mu}$, as

$$
\left(\begin{array}{c}
Z_{\mu}^{\prime} \\
X_{3 \mu}
\end{array}\right)=\left(\begin{array}{cc}
\cos \theta_{X}^{\prime} & -\sin \theta_{X}^{\prime} \\
\sin \theta_{X}^{\prime} & \cos \theta_{X}^{\prime}
\end{array}\right)\left(\begin{array}{c}
\tilde{Z}_{\mu}^{\prime} \\
\tilde{X}_{3 \mu}
\end{array}\right)
$$

with

$$
\tan \left(2 \theta_{X}^{\prime}\right)=\frac{2 c_{X} s_{X}}{c_{X}^{2}-\alpha s_{X}^{2}}
$$

we obtain the mass eigenvalues for dark gauge bosons,

$$
\begin{aligned}
& m_{\tilde{Z}^{\prime}}^{2}=m_{X_{3}}^{2} c_{X}^{2}\left(1-\cot \theta_{X}^{\prime} \tan \theta_{X}\right), \\
& m_{\tilde{X}_{3}}^{2}=m_{X_{3}}^{2} c_{X}^{2}\left(1+\tan \theta_{X}^{\prime} \tan \theta_{X}\right) .
\end{aligned}
$$

Therefore, from the results in eqs. (3.1)-(3.9), we can keep the hierarchy of masses,

$$
m_{X}^{2}<m_{\tilde{X}_{3}}^{2}<m_{\tilde{Z}^{\prime}}^{2},
$$

as far as

$$
\tan \theta_{X}^{\prime}<0, \quad\left|\tan \theta_{X}^{\prime}\right|<\frac{1}{2 \tan \theta_{X}} .
$$

In this case, the dark charged gauge boson is still the lightest gauge boson in the dark sector, so that the $2 \rightarrow 2$ annihilation of $X_{\mu}, X_{\mu}^{\dagger}$ is forbidden while $3 \rightarrow 2$ processes with gauge self-interactions become dominant for determining the relic density of $X_{\mu}, X_{\mu}^{\dagger}$.

We note that if $m_{X}>m_{\tilde{X}_{3}}$, the $2 \rightarrow 2$ annihilation of $X_{\mu}, X_{\mu}^{\dagger}$ into a $\tilde{X}_{3}$ pair is open, dominating the relic abundance calculation and leading to an interesting possibility for WIMP dark matter. However, in this work, we are interested in the production of light dark matter below sub-GeV scale, so henceforth we focus on the case with $m_{X}<m_{\tilde{X}_{3}}$.

Due to the mixing between $Z_{\mu}^{\prime}$ and $X_{3 \mu}$, self-interactions for $\mathrm{SU}(2)_{X}$ gauge bosons in eq. (2.6) become, in the basis of mass eigenstates,

$$
\mathcal{L}_{\text {self }}=\mathcal{L}_{3}+\mathcal{L}_{4}
$$

\footnotetext{
${ }^{2}$ In the presence of a gauge kinetic mixing between $Z^{\prime}$ and hypercharge gauge boson, mass eigenstates as well as mass eigenvalues of neutral gauge bosons including the $Z$-boson are modified, but we consider the case where mass corrections are negligible but new interactions of extra gauge bosons to the SM are kept in the leading order in the gauge kinetic mixing parameter, as will be discussed in the next section.
} 
with

$$
\begin{aligned}
\mathcal{L}_{3}= & -i g_{X} \cos \theta_{X}^{\prime}\left[\left(\partial^{\mu} X^{\nu}-\partial^{\nu} X^{\mu}\right) X_{\mu}^{\dagger} \tilde{X}_{3, \nu}-\left(\partial^{\mu} X^{\nu \dagger}-\partial^{\nu} X^{\mu \dagger}\right) X_{\mu} \tilde{X}_{3, \nu}\right. \\
& \left.+X_{\mu} X_{\nu}^{\dagger}\left(\partial^{\mu} \tilde{X}_{3}^{\nu}-\partial^{\nu} \tilde{X}_{3}^{\mu}\right)\right] \\
& -i g_{X} \sin \theta_{X}^{\prime}\left[\left(\partial^{\mu} X^{\nu}-\partial^{\nu} X^{\mu}\right) X_{\mu}^{\dagger} \tilde{Z}_{\nu}^{\prime}-\left(\partial^{\mu} X^{\nu \dagger}-\partial^{\nu} X^{\mu \dagger}\right) X_{\mu} \tilde{Z}_{\nu}^{\prime}\right. \\
& \left.+X_{\mu} X_{\nu}^{\dagger}\left(\partial^{\mu} \tilde{Z}^{\prime \nu}-\partial^{\nu} \tilde{Z}^{\prime \mu}\right)\right] \\
\mathcal{L}_{4}= & -\frac{g_{X}^{2}}{2}\left[\left(X_{\mu}^{\dagger} X^{\mu}\right)^{2}-X_{\mu}^{\dagger} X^{\mu \dagger} X_{\nu} X^{\nu}\right] \\
& -g_{X}^{2} \cos ^{2} \theta_{X}^{\prime}\left(X_{\mu}^{\dagger} X^{\mu} \tilde{X}_{\nu, 3} \tilde{X}_{3}^{\nu}-X_{\mu}^{\dagger} \tilde{X}_{3}^{\mu} X_{\nu} \tilde{X}_{3}^{\nu}\right) \\
& -g_{X}^{2} \sin ^{2} \theta_{X}^{\prime}\left(X_{\mu}^{\dagger} X^{\mu} \tilde{Z}_{\nu}^{\prime} \tilde{Z}^{\prime \nu}-X_{\mu}^{\dagger} \tilde{Z}^{\prime \mu} X_{\nu} \tilde{Z}^{\prime \nu}\right) \\
& -g_{X}^{2} \sin _{X}^{\prime} \cos \theta_{X}^{\prime}\left(2 X_{\mu}^{\dagger} X^{\mu} \tilde{X}_{3, \nu} \tilde{Z}^{\prime \nu}-X_{\mu}^{\dagger} \tilde{X}_{3}^{\mu} X_{\nu} \tilde{Z}^{\prime \nu}-X_{\mu}^{\dagger} \tilde{Z}^{\prime \mu} X_{\nu} \tilde{X}_{3}^{\nu}\right) .
\end{aligned}
$$

Moreover, in the basis of mass eigenstates for dark gauge bosons, for instance, from eq. (B.8) in the case of the triplet dark Higgs, we can also obtain the interactions between dark Higgs and gauge bosons. Concerning the self-interacting processes for dark-charged gauge bosons, we can use the above interactions by ignoring the mixing between dark Higgs and SM Higgs bosons and the mixings between dark-neutral gauge bosons and SM neutral gauge bosons.

\subsection{Split dark gauge bosons}

In the limit of $\alpha \gg 1$, the $Z^{\prime}$ boson decouples from $X$ and $\tilde{X}_{3}$. From eq. (3.7), we get $\tan \theta_{X}^{\prime} \approx-\frac{1}{\alpha \tan \theta_{X}}$, leading to the approximate gauge boson masses,

$$
\begin{aligned}
& m_{\tilde{Z}^{\prime}}^{2} \approx g_{X}^{2} I^{2} v_{I}^{2}\left(1+\alpha \tan ^{2} \theta_{X}\right), \\
& m_{\tilde{X}_{3}}^{2} \approx g_{X}^{2} I^{2} v_{I}^{2}\left(1-\frac{1}{\alpha}\right),
\end{aligned}
$$

leading to

$$
m_{\tilde{X}_{3}}^{2} \approx 2 \operatorname{Im}_{X}^{2}\left(1-\frac{1}{\alpha}\right)
$$

Then, in order for the dark-charged gauge boson $X$ to be a viable dark matter candidate, we require $m_{\tilde{X}_{3}}>m_{X}$, which means $I>\frac{1}{2}$, that is, at least a triplet Higgs field with nonzero VEV must be introduced. Moreover, in order for SIMP processes $X X X \rightarrow X \tilde{X}_{3}$ (which should be the dominant one in the relic abundance calculation) to be kinematically allowed, we also require $m_{\tilde{X}_{3}}<2 m_{X}$, which means $I \leq 2$. Thus, we need $m_{X}<m_{\tilde{X}_{3}}<2 m_{X}$ in order to realize a viable SIMP dark matter in our model.

From eq. (3.17), we get the general expression for the mass splitting parameter in the limit of large $\alpha$ :

$$
\begin{aligned}
\Delta \equiv \frac{m_{\tilde{X}_{3}}}{m_{X}}-1 & \approx \sqrt{2 I} \sqrt{1-\frac{1}{\alpha}}-1 \\
& \approx \sqrt{2 I}-1-\frac{\sqrt{2 I}}{2 \alpha},
\end{aligned}
$$


which will be relevant for forbidden channels in the later section. As a result, the mass relations between dark gauge bosons are dictated by dark custodial symmetry at the leading order, but up to small corrections due to the mixing with $Z^{\prime}$. For instance, we get $\Delta \approx \sqrt{2}-$ $1, \sqrt{3}-1,1$ for $I=1, \frac{3}{2}, 2$, respectively. Then, for the triplet Higgs case with $\Delta \approx \sqrt{2}-1$, we need to consider the $2 \rightarrow 2$ forbidden channels for dark matter annihilations. On the other hand, for quadruplet with $I=\frac{3}{2}$ or quintuplet with $I=2$, the mass splitting being too large, it is the $X X X \rightarrow X \tilde{X}_{3}$ process which is dominant. For general dark Higgs VEVs, we can make $\Delta$ to vary continuously from $\Delta \approx 0$ to $\Delta \approx 2$. We keep this fact in mind for scanning the parameter space for the correct relic density with general $X_{3}$ masses in the next section.

\subsection{Degenerate dark gauge bosons}

A quick look at eqs. (3.1) and (3.9) shows that there is a possibility of having degenerate mass terms in the dark sector, $m_{\tilde{X}_{3}} \approx m_{X}$ provided that $\tan \theta_{X}^{\prime} \approx-\frac{1}{2 I}(2 I-1) \cot \theta_{X}$ which gives, once combined with eq. (3.7): $\tan \theta_{X} \approx \frac{1}{\sqrt{2 I}} \sqrt{\frac{1}{\alpha-(2 I) /(2 I-1)}}$. Then, we obtain the abelian dark gauge boson mass as

$$
m_{\tilde{Z}^{\prime}}^{2} \approx 2 m_{X}^{2}\left(1+\tan ^{2} \theta_{X}\right)
$$

In this case, in the limit of $\tan \theta_{X} \gg 1$ or $g_{Z^{\prime}} \gg g_{X}$ for $\alpha \approx \frac{2 I}{2 I-1}>0$, we can have $\tilde{Z}^{\prime}$ decoupled. This case is possible for a nonzero VEV of the Higgs field with $I=1, \frac{3}{2}, 2$. Then, all the non-abelian gauge bosons of $\mathrm{SU}(2)$ can participate in the full $3 \rightarrow 2$ processes, without Boltzmann suppression. But, in this case, the mixing angle between dark neutral gauge bosons become suppressed due to $\left|\tan \theta_{X}^{\prime}\right| \ll 1$. Therefore, DM-SM elastic scattering through the kinetic mixing would be suppressed, so we need to rely on Higgs portal coupling for kinetic equilibrium.

If one considers multiple Higgs fields with different isospins, in particular, doublet $\Phi$ and triplet $T$, from eq. (A.11), we get the approximate mass difference in the limit of a large $\tilde{Z}^{\prime}$ mass:

$$
m_{\tilde{X}_{3}}^{2}-m_{X}^{2} \approx \frac{1}{2} g_{X}^{2} v_{T}^{2}-\frac{1}{\beta} g_{X}^{2}\left(\frac{1}{4} v_{\Phi}^{2}+v_{T}^{2}\right)
$$

with

$$
\beta=1+\frac{q_{S}^{2} v_{S}^{2}}{\frac{1}{2} v_{\Phi}^{2}+v_{T}^{2}} \gg 1 .
$$

Then, we can tune dark Higgs VEVs, $v_{\Phi}$ and $v_{T}$ such that $m_{\tilde{X}_{3}}^{2} \approx m_{X}^{2}$. In this case, as discussed in the appendix, the mixing angle between dark neutral gauge bosons becomes

$$
\tan \theta_{X}^{\prime} \approx-\frac{1}{\beta \tan \theta_{X}} .
$$

Therefore, even in this case, the mixing angle in the dark gauge sector is small so Higgs portal coupling would be more relevant for kinetic equilibrium as in the previous case. 


\section{Dark matter annihilations with self-interactions}

We consider the Boltzmann equations for determining the dark matter relic density mainly by the DM self-interactions, in particular, by taking $3 \rightarrow 2$ channels and forbidden $2 \rightarrow 2$ channels to be dominant. We also present the results on DM self-scattering cross sections and impose current astrophysical limits on them together with the condition for a correct relic density.

\subsection{Boltzmann equations}

For $C P$ conserving interactions of dark matter, we get $n_{X^{+}}=n_{X^{-}}$. Then, the total DM number density, $n_{X}=n_{X^{+}}+n_{X^{-}}$, is governed by the following Boltzmann equation,

$$
\begin{aligned}
\dot{n}_{X}+3 H n_{X}= & -\left\langle\sigma v_{\text {rel }}\right\rangle_{2 \rightarrow 2, v}\left(n_{X}^{2}-n_{X, \text { eq }}^{2}\right)-\left\langle\sigma v_{\text {rel }}\right\rangle_{3 \rightarrow 2}\left(n_{X}^{3}-n_{X} n_{X, \text { eq }}^{2}\right) \\
& +\left\langle\sigma v_{\text {rel }}\right\rangle_{2 \rightarrow 2, d} n_{\tilde{X}_{3}, \text { eq }}^{2}\left(1-\frac{n_{X}^{2}}{n_{X, \text { eq }}^{2}}\right)
\end{aligned}
$$

where

$$
\begin{aligned}
\left\langle\sigma v_{\text {rel }}\right\rangle_{2 \rightarrow 2, v} & \equiv \frac{1}{2}\left\langle\sigma v_{\text {rel }}\right\rangle_{X_{+} X_{-} \rightarrow f \bar{f}}, \\
\left\langle\sigma v_{\text {rel }}\right\rangle_{3 \rightarrow 2} & \equiv \frac{1}{2}\left\langle\sigma v_{\mathrm{rel}}^{2}\right\rangle_{X_{+} X_{+} X_{-} \rightarrow X_{+} \tilde{X}_{3}}, \\
\left\langle\sigma v_{\text {rel }}\right\rangle_{2 \rightarrow 2, d} & \equiv 2\left\langle\sigma v_{\text {rel }}\right\rangle_{\tilde{X}_{3} \tilde{X}_{3} \rightarrow X_{+} X_{-}} .
\end{aligned}
$$

Here, in the last line on right in eq. (4.1), we have already used the detailed balance condition for forbidden channels to obtain the cross section for forbidden $2 \rightarrow 2$ annihilation, $X_{+} X_{-} \rightarrow \tilde{X}_{3} \tilde{X}_{3}$, as follows,

$$
\left\langle\sigma v_{\mathrm{rel}}\right\rangle_{X_{+} X_{-} \rightarrow \tilde{X}_{3} \tilde{X}_{3}}=\frac{4 n_{\tilde{X}_{3}, \mathrm{eq}}^{2}}{n_{X, \mathrm{eq}}^{2}}\left\langle\sigma v_{\mathrm{rel}}\right\rangle_{\tilde{X}_{3} \tilde{X}_{3} \rightarrow X_{+} X_{-}} .
$$

Here, we assumed that $\tilde{X}_{3}$ keeps in thermal equilibrium with the SM during freeze-out, and the equilibrium abundances for $X$ and $\tilde{X}_{3}$ are given by

$$
Y_{X, \mathrm{eq}}=\frac{n_{X, \mathrm{eq}}}{s}=\frac{45 x^{2}}{2 g_{* s} \pi^{4}} K_{2}(x), \quad Y_{\tilde{X}_{3}, \mathrm{eq}}=\frac{n_{\tilde{X}_{3}, \mathrm{eq}}}{s}=\frac{45 m_{\tilde{X}_{3}}^{2} x^{2}}{4 g_{* s} \pi^{4} m_{X}^{2}} K_{2}\left(\frac{m_{\tilde{X}_{3}} x}{m_{X}}\right)
$$

with $x \equiv m_{X} / T$. We have also included the SM $2 \rightarrow 2$ annihilations, $X_{+} X_{-} \rightarrow f \bar{f}$, in the Boltzmann equation (4.1), as will be discussed in the next section.

\section{$4.2 \quad 3 \rightarrow 2$ annihilations}

The effective $3 \rightarrow 2$ annihilation cross section in eq. (4.1) is given by the channel, $X_{+} X_{+} X_{-} \rightarrow X_{+} \tilde{X}_{3}$, as shown in figure 1 , with the corresponding cross section given by

$$
\begin{aligned}
\left\langle\sigma v^{2}\right\rangle_{X_{+} X_{+} X_{-} \rightarrow X_{+} \tilde{X}_{3}}= & \frac{\cos ^{2}\left(\theta_{X}^{\prime}\right) g_{X}^{6}}{161243136 \pi m_{X}^{5}}\left(\frac{m_{\tilde{X}_{3}}}{m_{X}}\right)^{14}\left(1-\frac{m_{\tilde{X}_{3}}^{2}}{16 m_{X}^{2}}\right)^{3 / 2}\left(1-\frac{m_{\tilde{X}_{3}}^{2}}{4 m_{X}^{2}}\right)^{-1 / 2} \\
& \times\left(1+\frac{m_{\tilde{X}_{3}}^{2}}{2 m_{X}^{2}}\right)^{-2}\left(3 A_{1}+4 \cos \left(2 \theta_{X}^{\prime}\right) A_{2}+\cos \left(4 \theta_{X}^{\prime}\right) A_{3}\right)
\end{aligned}
$$



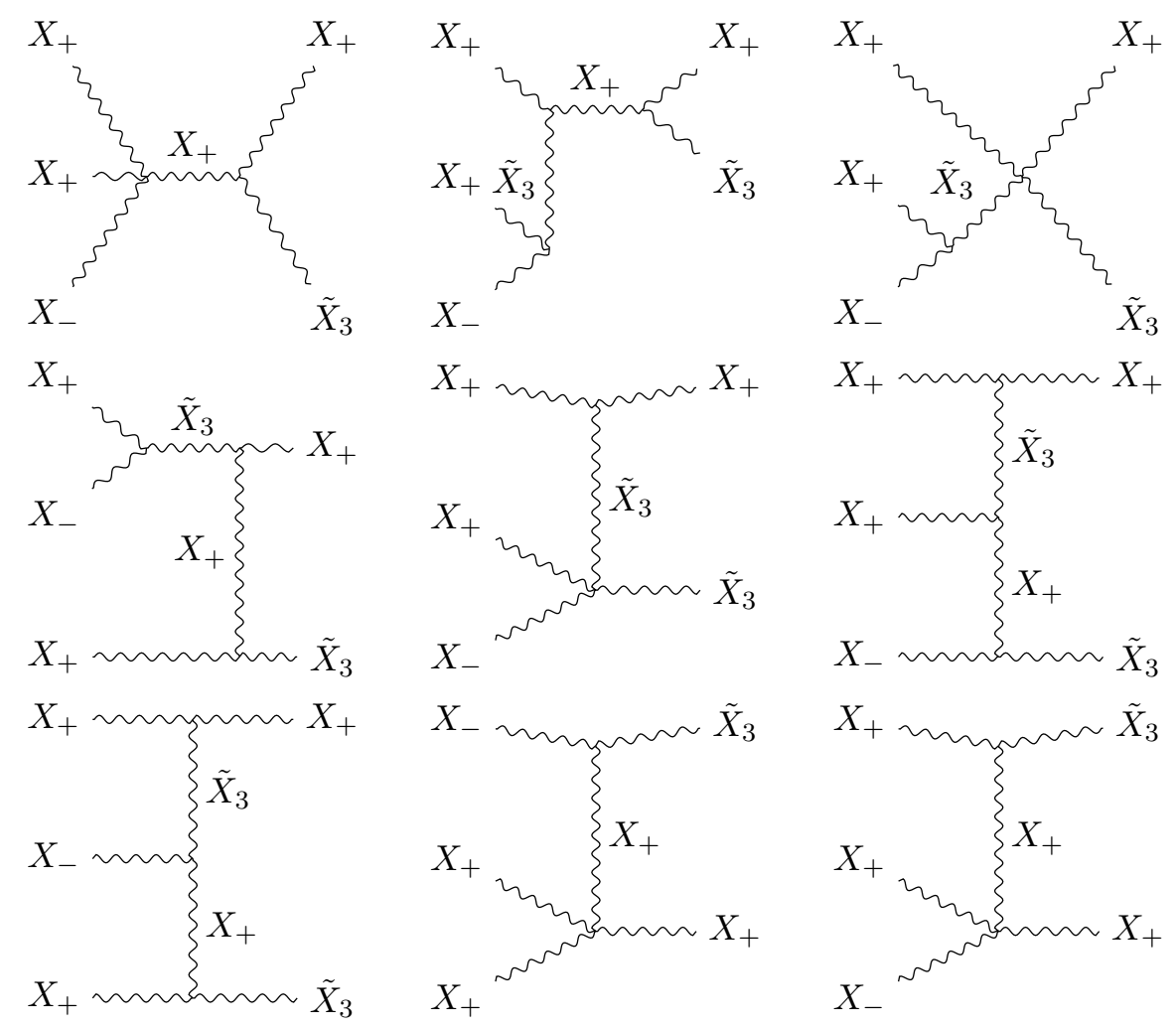

Figure 1. Feynman diagrams for $X_{+} X_{+} X_{-} \rightarrow X_{+} \tilde{X}_{3}$.

where

$$
\begin{aligned}
A_{1}= & 17+823\left(\frac{m_{X}}{m_{\tilde{X}_{3}}}\right)^{2}+5380\left(\frac{m_{X}}{m_{\tilde{X}_{3}}}\right)^{4}+306672\left(\frac{m_{X}}{m_{\tilde{X}_{3}}}\right)^{6}-1964704\left(\frac{m_{X}}{m_{\tilde{X}_{3}}}\right)^{8} \\
& +6233600\left(\frac{m_{X}}{m_{\tilde{X}_{3}}}\right)^{10}-3080192\left(\frac{m_{X}}{m_{\tilde{X}_{3}}}\right)^{12}+13860864\left(\frac{m_{X}}{m_{\tilde{X}_{3}}}\right)^{14}, \\
A_{2}= & +219\left(\frac{m_{X}}{m_{\tilde{X}_{3}}}\right)^{2}+180\left(\frac{m_{X}}{m_{\tilde{X}_{3}}}\right)^{4}+69152\left(\frac{m_{X}}{m_{\tilde{X}_{3}}}\right)^{6}-1351488\left(\frac{m_{X}}{m_{\tilde{X}_{3}}}\right)^{8} \\
& +6657120\left(\frac{m_{X}}{m_{\tilde{X}_{3}}}\right)^{10}-8880832\left(\frac{m_{X}}{m_{\tilde{X}_{3}}}\right)^{12}+9142272\left(\frac{m_{X}}{m_{\tilde{X}_{3}}}\right)^{14}, \\
A_{3}= & +39\left(\frac{m_{X}}{m_{\tilde{X}_{3}}}\right)^{2}-228\left(\frac{m_{X}}{m_{\tilde{X}_{3}}}\right)^{4}+12400\left(\frac{m_{X}}{m_{\tilde{X}_{3}}}\right)^{6}-399648\left(\frac{m_{X}}{m_{\tilde{X}_{3}}}\right)^{8} \\
& +3725184\left(\frac{m_{X}}{m_{\tilde{X}_{3}}}\right)^{10}-12369536\left(\frac{m_{X}}{m_{\tilde{X}_{3}}}\right)^{12}+22413312\left(\frac{m_{X}}{m_{\tilde{X}_{3}}}\right)^{14} .
\end{aligned}
$$

Then, assuming that dark matter annihilates mainly due to $3 \rightarrow 2$ channels, we can determine the relic density [51-53] as

$$
\Omega_{X} h^{2} \simeq 0.12\left(\frac{g_{*}}{10.75}\right)^{-3 / 4}\left(\frac{x_{f}}{15}\right)^{2}\left(\frac{m_{X} / \alpha_{\mathrm{eff}}}{45 \mathrm{MeV}}\right)^{3 / 2}
$$

where $\alpha_{\text {eff }}$ is defined from $\left\langle\sigma v_{\text {rel }}^{2}\right\rangle_{3 \rightarrow 2} \equiv \alpha_{\text {eff }}^{3} / m_{X}^{5}$. 


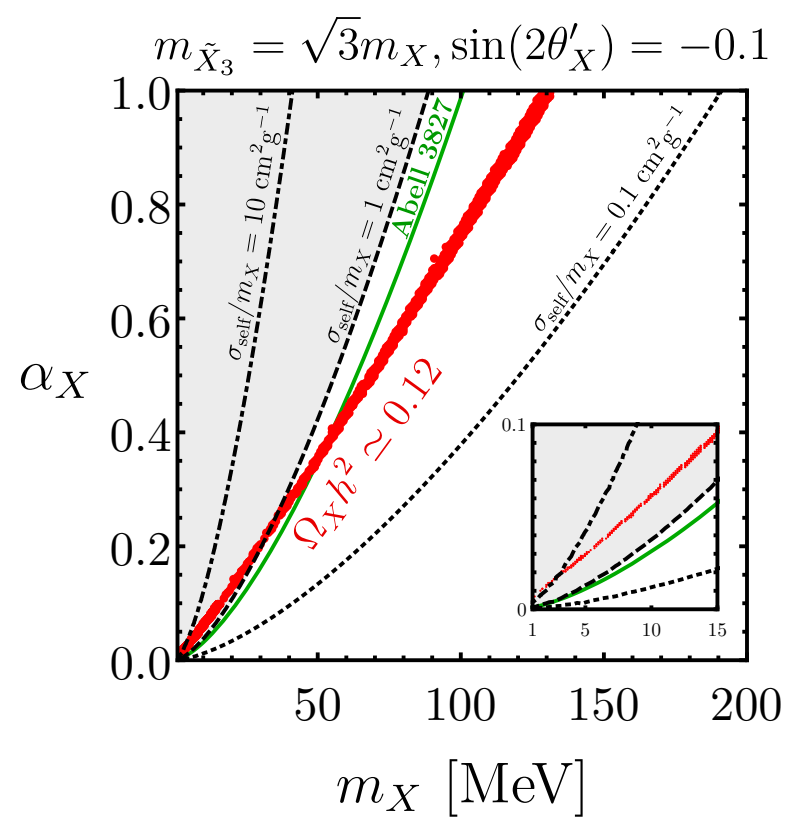

Figure 2. Parameter space for $m_{X}$ vs $\alpha_{X} \equiv g_{X}^{2} / 4 \pi$ for the case with quadruplet dark Higgs. The correct relic density is satisfied in red region. Contours for DM self-scattering cross section with $\sigma_{\text {self }} / m_{X}=0.1,1,10 \mathrm{~cm}^{2} / \mathrm{g}$ are shown in dotted, dashed and dot-dashed lines, respectively. We also indicated the favored parameter space in green line to explain the off-set of one of the galaxies in Abell 3827 [71-73] and the region disfavored by Bullet cluster in gray [74-76].

In figure 2, we showed the parameter space for $m_{X}$ and $\alpha_{X} \equiv g_{X}^{2} /(4 \pi)$, satisfying the relic density condition in red region, dominantly due to SIMP channels. We took the VEV of quadruplet Higgs to get masses for non-abelian gauge bosons, for which $m_{\tilde{X}_{3}} \approx \sqrt{3} m_{X}$. Forbidden channels become dominant only for small $m_{X}$ or $\alpha_{X}$. We also drew the contours with $\sigma_{\text {self }} / m_{X}=0.1,1,10 \mathrm{~cm}^{2} / \mathrm{g}$ in dotted, dashed and dot-dashed lines. We note that the observed off-set of one of the galaxies of Abell 3827 [71, 72] might indicate a large self-scattering cross section for dark matter, $\sigma_{\text {self }} \cos \theta_{i} / m_{X} \sim 0.68 \mathrm{~cm}^{2} / \mathrm{g}$ [73], where the cross section value depends on the unknown inclination angle $\theta_{i}$ of the galaxy's 3D motion with respect to the plane of the sky. For comparison, taking $\theta_{i}=0$, we also showed the parameter space potentially favored by Abell 3827 in green line. The gray region is disfavored by Bullet cluster bound [74-76], $\sigma_{\text {self }} / m_{X} \lesssim 1 \mathrm{~cm}^{2} / \mathrm{g}$.

In figure 3 , we also depicted the parameter space for $m_{X}$ and $m_{\tilde{X}_{3}}$, satisfying the correct relic density in red regions. We took different values for $\alpha_{X}=0.1,0.5,1$, from left to right. We included both the SIMP and forbidden $2 \rightarrow 2$ channels for determining the relic density. Forbidden channels are important for $m_{\tilde{X}_{3}} \lesssim 1.6 m_{X}$, as will be discussed in the next subsection. In each plot of figure 3, the SIMP channels are kinematically closed in the upper purple region because $m_{\tilde{X}_{3}}>2 m_{X}$, whereas the $2 \rightarrow 2$ annihilations such as $X_{+} X_{-} \rightarrow \tilde{X}_{3} \tilde{X}_{3}$ are open and dominant in the lower green region. For comparison, the mass relation, $m_{\tilde{X}_{3}} \approx \sqrt{3} m_{X}$, is satisfied along the blue dashed line. Below the blue dashed line, the forbidden channels tend to contribute to the DM annihilations dominantly. 

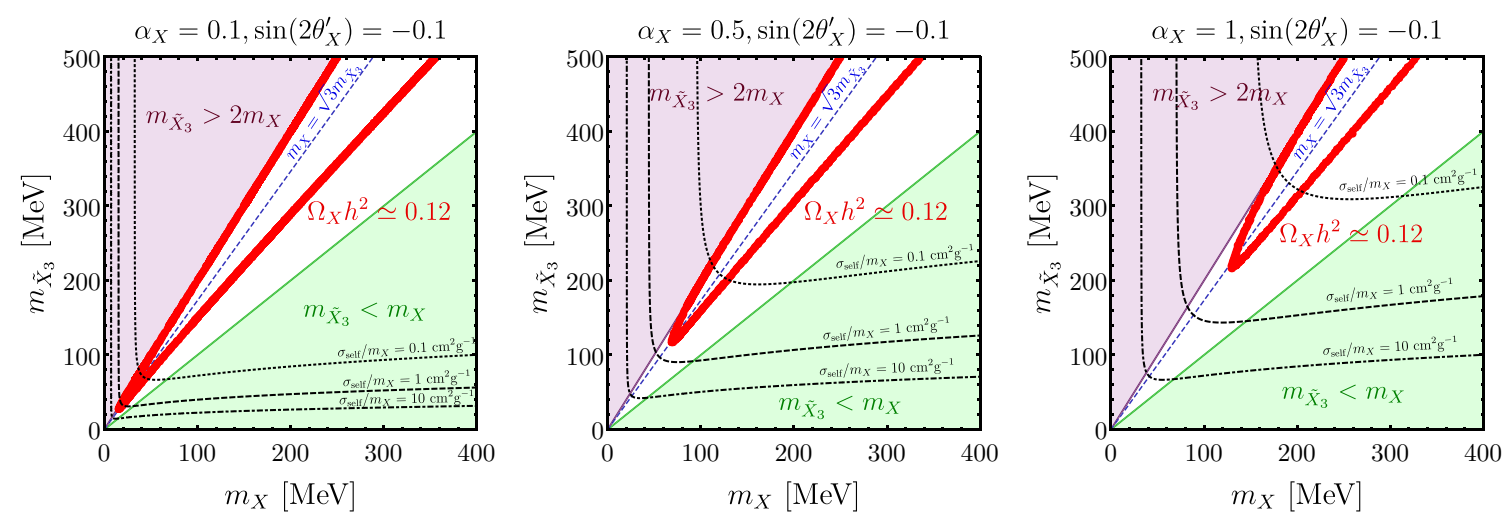

Figure 3. Parameter space for $m_{X}$ vs $m_{\tilde{X}_{3}}$. Both SIMP and forbidden $2 \rightarrow 2$ channels are included for determining the relic density. Contours with DM self-scattering cross section, $\sigma_{\text {self }} / m_{X}=$ $0.1,1,10 \mathrm{~cm}^{2} / \mathrm{g}$ are shown in dotted, dashed and dotdashed lines, respectively. SIMP processes are not allowed kinematically in the upper purple region whereas $2 \rightarrow 2$ annihilations in the dark sector are dominant in the lower green region. The blue dashed line corresponds to the mass relation, $m_{\tilde{X}_{3}}=\sqrt{3} m_{X}$, for the quadruplet dark Higgs.

But, above the blue dashed line, the SIMP channels are dominant, and the relic density is saturated close to $m_{\tilde{X}_{3}} \sim 2 m_{X}$, due to the $t$-channel diagrams. This behavior is regularized by a relatively large velocity of dark matter during freeze-out.

\subsection{Forbidden $2 \rightarrow 2$ annihilations}

Next, the forbidden $2 \rightarrow 2$ annihilation channels are shown in figure 4 . For the inverse process of $2 \rightarrow 2$ annihilation, i.e. $\tilde{X}_{3} \tilde{X}_{3} \rightarrow X_{+} X_{-}$, in the dark sector, the corresponding cross section is given by

$$
\begin{aligned}
\left\langle\sigma v_{\text {rel }}\right\rangle_{\tilde{X}_{3} \tilde{X}_{3} \rightarrow X_{+} X_{-}}= & \frac{\cos ^{4}\left(\theta_{X}^{\prime}\right) g_{X}^{4}}{144 \pi m_{X}^{2}}\left(\frac{m_{\tilde{X}_{3}}}{m_{X}}\right)^{6} \sqrt{1-\frac{m_{X}^{2}}{m_{\tilde{X}_{3}^{2}}}} \\
\times & {\left[1-2\left(\frac{m_{X}}{m_{\tilde{X}_{3}}}\right)^{2}+\left(\frac{m_{X}}{m_{\tilde{X}_{3}}}\right)^{4}+64\left(\frac{m_{X}}{m_{\tilde{X}_{3}}}\right)^{6}-71\left(\frac{m_{X}}{m_{\tilde{X}_{3}}}\right)^{8}\right.} \\
& \left.+88\left(\frac{m_{X}}{m_{\tilde{X}_{3}}}\right)^{10}+48\left(\frac{m_{X}}{m_{\tilde{X}_{3}}}\right)^{12}\right]
\end{aligned}
$$

For dominance with forbidden channels in the Boltzmann equation (4.1), the solution for the DM abundance $[52,53]$ becomes

$$
Y_{X}(\infty)=\frac{x_{f}}{\lambda} e^{2 \Delta x_{f}} f\left(\Delta, x_{f}\right)
$$

where $\lambda \equiv s\left(m_{X}\right) / H\left(m_{X}\right)$ with $s\left(m_{X}\right)$ and $H\left(m_{X}\right)$ being entropy density and Hubble parameter, respectively, evaluated at $T=m_{X}$, and

$$
f\left(\Delta, x_{f}\right)=\left[\frac{1}{2}\left\langle\sigma v_{\mathrm{rel}}\right\rangle_{\tilde{X}_{3} \tilde{X}_{3} \rightarrow X_{+} X_{-}}(1+\Delta)^{3}\left(1-2 \Delta x_{f} e^{2 \Delta x_{f}} \int_{2 \Delta x_{f}}^{\infty} d t t^{-1} e^{-t}\right)\right]^{-1}
$$



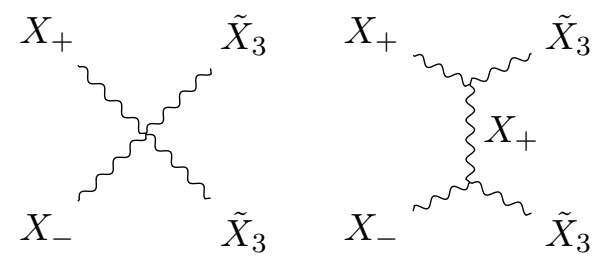

Figure 4. Feynman diagrams for $X_{+} X_{-} \rightarrow \tilde{X}_{3} \tilde{X}_{3}$.
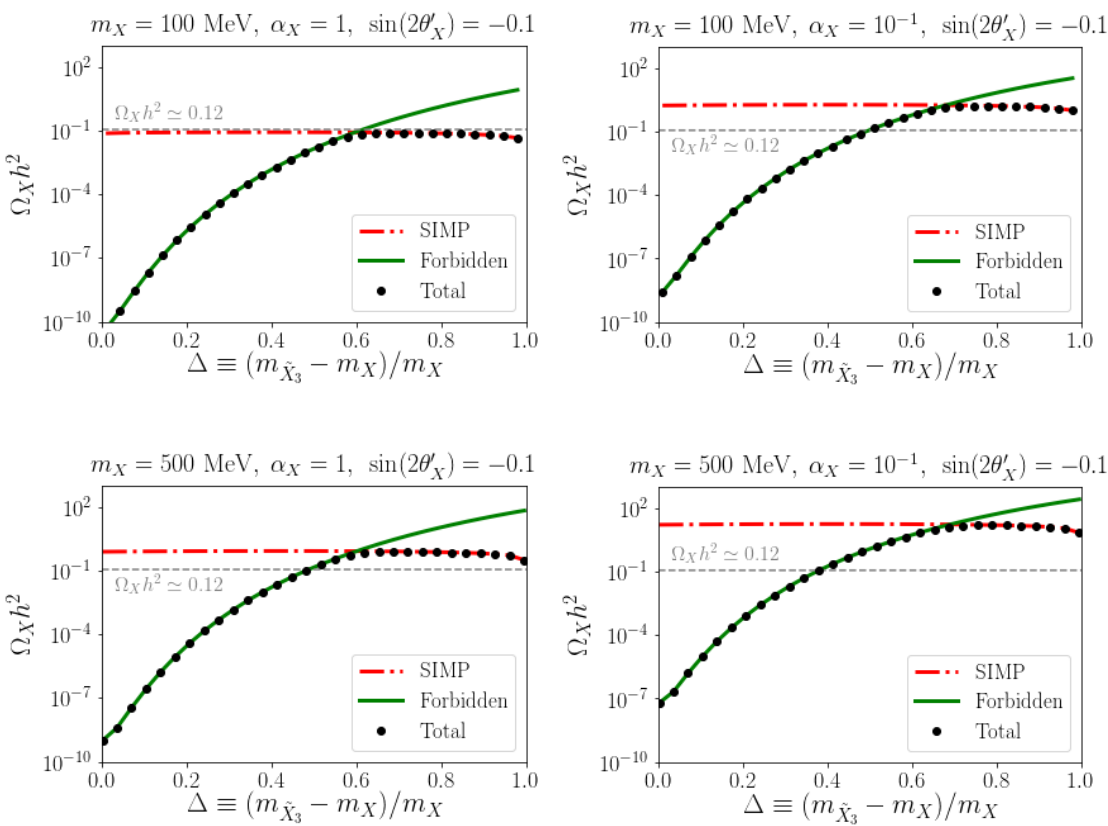

Figure 5. Relic density as a function of $\Delta=\left(m_{\tilde{X}_{3}}-m_{X}\right) / m_{X}$. SIMP channels only are in red and forbidden channels only are in green, while both channels are included in black.

and $\Delta \equiv\left(m_{\tilde{X}_{3}}-m_{X}\right) / m_{X}$. Then, forbidden channels can determine the relic abundance $[52,53]$ as follows,

$$
\Omega_{X} h^{2} \simeq 0.12\left(\frac{g_{*}}{10.75}\right)^{-1 / 2}\left(\frac{x_{f}}{15}\right) e^{2(\Delta-0.6) x_{f}}\left(\frac{m_{X}}{100 \mathrm{MeV}}\right)^{2}\left(\frac{4.6 \times 10^{-2}}{\beta_{\mathrm{eff}}}\right)^{2}
$$

where $\beta_{\text {eff }}$ is defined from $f\left(\Delta, x_{f}\right) \equiv m_{X}^{2} / \beta_{\text {eff }}^{2}$ and we took $x_{f} \simeq 15$ in the exponent. As a consequence, as far as $\Delta \lesssim 0.6$, the forbidden channels can be efficient enough even for a small self-interaction to produce a correct relic density. This is the case with the triplet dark Higgs, for which $\Delta \approx \sqrt{2}-1$. In cases with quadruplet and quintuplet dark Higgs fields, $\Delta$ is larger so that SIMP channels are dominant in determining the relic density.

In figure 5, we showed the relic density as a function of the mass difference, $\Delta=\left(m_{\tilde{X}_{3}}-m_{X}\right) / m_{X}$ in black dotted line. In each plot, SIMP channels only are assumed in red line and forbidden channels only are assumed in green line. We have included both SIMP and forbidden channels in black line. In all the cases for $m_{X} \sim 100-500 \mathrm{MeV}$, SIMP channels become dominant for $\Delta \gtrsim 0.6$ or $m_{\tilde{X}_{3}} \gtrsim 1.6 m_{X}$. Therefore, from eq. (3.18), $\Delta \simeq \sqrt{2 I}-1$, so we need that dark Higgs fields with $I=\frac{3}{2}$ or 2 determine the $\operatorname{SU}(2)_{X}$ 

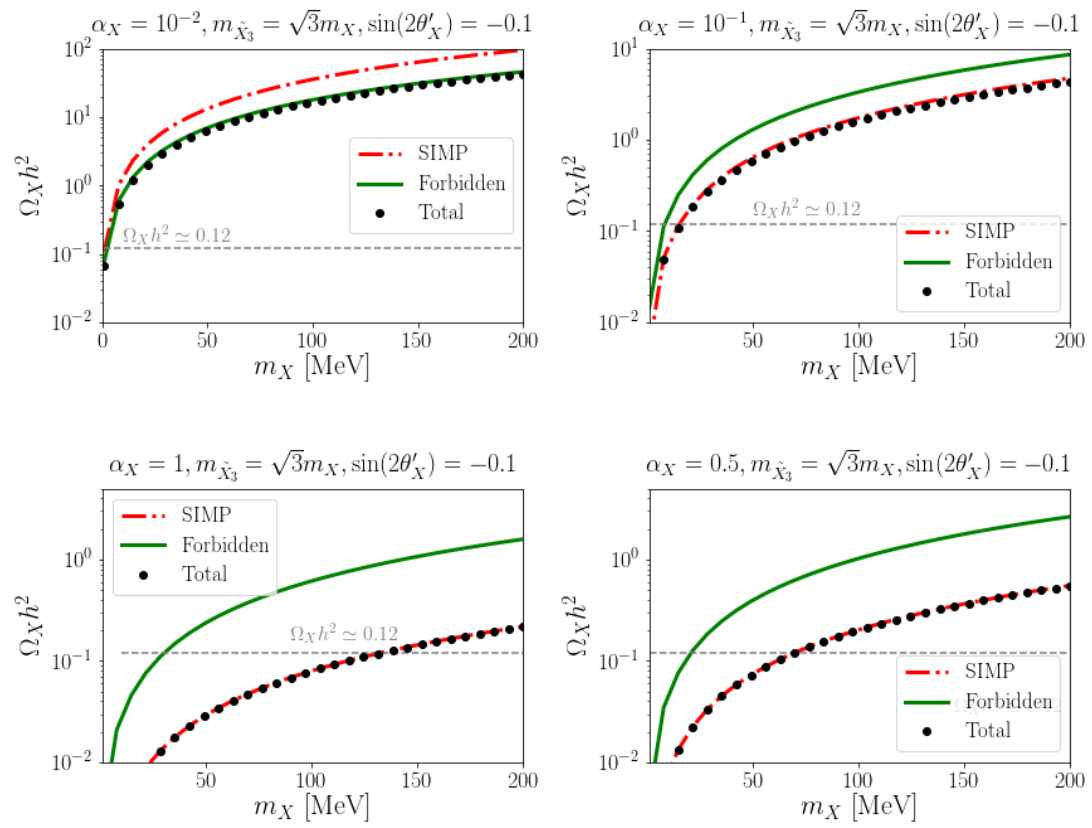

Figure 6. Relic density as a function of $m_{X}$ for the case with quadruplet dark Higgs. SIMP channels only are in red and forbidden channels only are in green, while both channels are included in black.

gauge boson masses dominantly. Otherwise, the relic density is determined mainly by forbidden channels.

In figure 6 , we drew similar plots as in figure 5 , but now for the relic density as a function of $m_{X}$ for the fixed mass relation, $m_{\tilde{X}_{3}}=\sqrt{3} m_{X}$, for the nonzero VEV of quadruplet Higgs. In this case, as we increase $\alpha_{X}$ from 0.01 to $0.1,0.5$ and 1, clockwise, showing the dominance of SIMP channels in determining the relic density for $\alpha_{X} \gtrsim 0.1$. For a correct relic density, we need a sizable $\alpha_{X}$ as in the plots in the lower panel, in which case the SIMP channels are dominant.

\subsection{DM self-scattering}

The DM $2 \rightarrow 2$ self-scattering channels are given in figure 7 . Then, the effective DM self-scattering cross section is given by

$$
\begin{aligned}
\sigma_{\text {self }} & =\frac{1}{4}\left(\sigma_{X_{+} X_{-} \rightarrow X_{+} X_{-}}+\sigma_{X_{+} X_{+} \rightarrow X_{+} X_{+}}+\sigma_{X_{-} X_{-} \rightarrow X_{-} X_{-}}\right) \\
& =\frac{g_{X}^{4}}{96 \pi m_{X}^{2}}\left[3+2\left(\frac{m_{X}}{m_{\tilde{X}_{3}}} \cos \left(\theta_{X}^{\prime}\right)\right)^{2}+22\left(\frac{m_{X}}{m_{\tilde{X}_{3}}} \cos \left(\theta_{X}^{\prime}\right)\right)^{4}\right]
\end{aligned}
$$

with

$$
\begin{aligned}
\sigma_{X_{+} X_{+} \rightarrow X_{+} X_{+}} & =\sigma_{X_{-} X_{-} \rightarrow X_{-} X_{-}} \\
& =\frac{g_{X}^{4}}{96 \pi m_{X}^{2}}\left[3+8\left(\frac{m_{X}}{m_{\tilde{X}_{3}}} \cos \left(\theta_{X}^{\prime}\right)\right)^{2}+32\left(\frac{m_{X}}{m_{\tilde{X}_{3}}} \cos \left(\theta_{X}^{\prime}\right)\right)^{4}\right], \\
\sigma_{X_{+} X_{-} \rightarrow X_{+} X_{-}} & =\frac{g_{X}^{4}}{48 \pi m_{X}^{2}}\left[3-4\left(\frac{m_{X}}{m_{\tilde{X}_{3}}} \cos \left(\theta_{X}^{\prime}\right)\right)^{2}+12\left(\frac{m_{X}}{m_{\tilde{X}_{3}}} \cos \left(\theta_{X}^{\prime}\right)\right)^{4}\right] .
\end{aligned}
$$




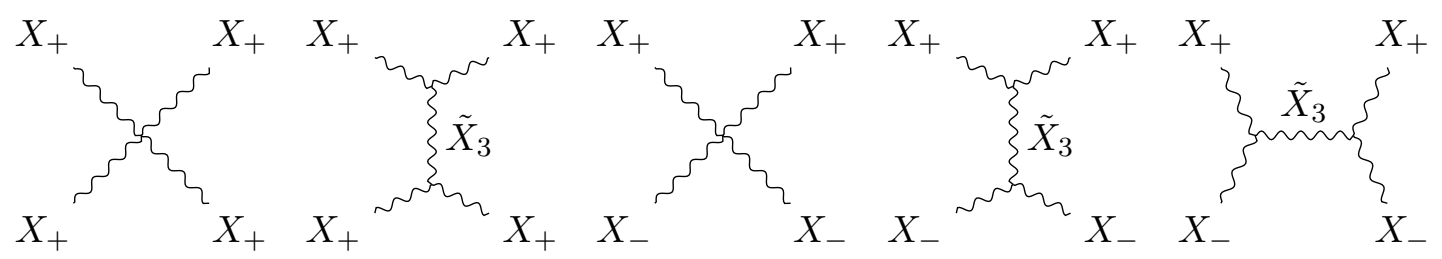

Figure 7. Feynman diagrams for DM self-scattering channels, $X_{ \pm} X_{ \pm} \rightarrow X_{ \pm} X_{ \pm}$and $X_{+} X_{-} \rightarrow X_{+} X_{-}$.

Then, the DM self-scattering with $\sigma_{\text {self }} / m_{X}=0.1-10 \mathrm{~cm}^{2} / \mathrm{g}$ can explain small-scale problems at galaxies such as core-cusp problem, too-big-to-fail problem, etc. On the other hand, we also note that the DM self-scattering cross section is constrained to be $\sigma_{\text {self }} / m_{X} \lesssim 1 \mathrm{~cm}^{2} / \mathrm{g}$ by either Bullet cluster [74-76] or halo shapes/ellipticity [77].

\section{$5 \quad Z^{\prime}$ portal couplings for dark matter}

Dark matter, if its abundance is determined mainly by SIMP processes, must be in kinetic equilibrium with another species in the dark or visible sectors for the structure formation. In order not to introduce the temperature of dark matter as an independent parameter, we consider the case that dark matter is equilibriated by the elastic scattering between dark matter and light particles in the SM.

In this section, we discuss the conditions for kinetic equilibrium in the presence of a gauge kinetic mixing between $Z^{\prime}$ and SM hypercharge gauge bosons, and consider various constraints on the model, coming from the consistency of SIMP scenarios to experimental bounds such as direct detection and collider searches.

\subsection{General current interactions with $Z^{\prime}$ portal}

In the presence of a gauge kinetic mixing, we need to consider the full basis of neutral gauge bosons, including neutral dark gauge bosons and those in the SM, i.e. $B_{\mu}$ and $W_{3 \mu}$. For a zero dark Weinberg angle, we can easily diagonalize the gauge kinetic terms and mass terms for neutral gauge bosons only by the $4 \times 4$ rotation matrix, $O_{W}$, as in usual $Z^{\prime}$ portal models. Even with a nonzero dark Weinberg angle, we can still diagonalize the mass matrix by approximate rotations with $O_{W}$, followed by the $2 \times 2$ rotation matrix, $O_{X}$, in the limit of a small gauge kinetic mixing. Then, the gauge bosons in the interaction basis, $\left(B_{\mu}, W_{3 \mu}, Z_{\mu}^{\prime}, X_{3 \mu}\right)$, can be written in terms of approximate mass eigenstates $\left(\tilde{A}_{\mu}, \tilde{Z}_{\mu}, \tilde{Z}_{\mu}^{\prime}, \tilde{X}_{3 \mu}\right)$, as follows,

$$
\left(\begin{array}{c}
B_{\mu} \\
W_{3 \mu} \\
Z_{\mu}^{\prime} \\
X_{3 \mu}
\end{array}\right)=O_{W} O_{X}\left(\begin{array}{c}
\tilde{A}_{\mu} \\
\tilde{Z}_{\mu} \\
\tilde{Z}_{\mu}^{\prime} \\
\tilde{X}_{3 \mu}
\end{array}\right)
$$


with

$$
\begin{aligned}
O_{W} & =\left(\begin{array}{cccc}
c_{W} & t_{\xi} s_{\zeta}-s_{W} c_{\zeta}-s_{W} s_{\zeta}-t_{\xi} c_{\zeta} & 0 \\
s_{W} & c_{W} c_{\zeta} & c_{W} s_{\zeta} & 0 \\
0 & -s_{\zeta} / c_{\xi} & c_{\zeta} / c_{\xi} & 0 \\
0 & 0 & 0 & 1
\end{array}\right), \\
O_{X} & =\left(\begin{array}{cccc}
1 & 0 & 0 & 0 \\
0 & 1 & 0 & 0 \\
0 & 0 & c_{\theta_{X}^{\prime}} & -s_{\theta_{X}^{\prime}} \\
0 & 0 & s_{\theta_{X}^{\prime}} & c_{\theta_{X}^{\prime}}^{\prime}
\end{array}\right)
\end{aligned}
$$

Here, $c_{\xi} \equiv \cos \xi, t_{\xi} \equiv \tan \xi, c_{W} \equiv \cos \theta_{W}, s_{W} \equiv \sin \theta_{W}$, and $\zeta$ is the approximate mixing angle between $Z^{\prime}$ and $Z$ bosons, given [50] by

$$
\tan (2 \zeta)=\frac{m_{Z}^{2} s_{W} \sin (2 \xi)}{m_{Z^{\prime}}^{2}-m_{Z}^{2}\left(c_{\xi}^{2}-s_{W}^{2} s_{\xi}^{2}\right)}
$$

In the limit of $m_{Z^{\prime}}^{2} \ll m_{Z}^{2}$ and $|\xi| \ll 1$, we obtain $\zeta \approx-s_{W} \xi$. Moreover, the approximate mass eigenvalues for $Z$-like and $Z^{\prime}$-like gauge bosons are given [50] by

$$
m_{1,2}^{2}=\frac{1}{2}\left[m_{Z}^{2}\left(1+s_{W}^{2} t_{\xi}^{2}\right)+m_{Z^{\prime}}^{2} / c_{\xi}^{2} \pm \sqrt{\left(m_{Z}^{2}\left(1+s_{W}^{2} t_{\xi}^{2}\right)+m_{Z^{\prime}}^{2} / c_{\xi}^{2}\right)^{2}-4 m_{Z^{2}}^{2} m_{Z^{\prime}}^{2} / c_{\xi}^{2}}\right] .
$$

The current interactions in the interaction basis are given by

$$
\begin{aligned}
\mathcal{L}_{\mathrm{EM} / \mathrm{NC}}= & g_{X} X_{3 \mu} J_{X_{3}}^{\mu}+g_{Z^{\prime}} Z_{\mu}^{\prime} J_{Z^{\prime}}^{\mu} \\
& +e\left(s_{W} W_{3 \mu}+c_{W} B_{\mu}\right) J_{\mathrm{EM}}^{\mu}+\frac{e}{2 s_{W} c_{W}}\left(c_{W} W_{3 \mu}-s_{W} B_{\mu}\right) J_{Z}^{\mu}
\end{aligned}
$$

where $J_{X_{3}}^{\mu}$ and $J_{Z^{\prime}}^{\mu}$ are dark-neutral and $Z^{\prime}$ currents, and $J_{\mathrm{EM}}^{\mu}$ and $J_{Z}^{\mu}$ are electromagnetic and neutral currents. Then, using eq. (5.1), we can rewrite the above current interactions in the basis of mass eigenstates, at first order in $\varepsilon \equiv c_{W} t_{\xi} \approx c_{W} \xi$, as

$$
\begin{aligned}
\mathcal{L}_{\mathrm{EM} / \mathrm{NC}}= & e \tilde{A}_{\mu} J_{\mathrm{EM}}^{\mu}+\tilde{Z}_{\mu}\left[\frac{e}{2 s_{W} c_{W}} J_{Z}^{\mu}+\varepsilon g_{Z^{\prime}} t_{W} J_{Z^{\prime}}^{\mu}\right] \\
& +\tilde{Z}_{\mu}^{\prime}\left[g_{X} \sin \left(\theta_{X}^{\prime}\right) J_{X_{3}}^{\mu}+g_{Z^{\prime}} \cos \left(\theta_{X}^{\prime}\right) J_{Z^{\prime}}^{\mu}-e \varepsilon \cos \left(\theta_{X}^{\prime}\right) J_{\mathrm{EM}}^{\mu}\right] \\
& +\tilde{X}_{3 \mu}\left[g_{X} \cos \left(\theta_{X}^{\prime}\right) J_{X_{3}}^{\mu}-g_{Z^{\prime}} \sin \left(\theta_{X}^{\prime}\right) J_{Z^{\prime}}^{\mu}+e \varepsilon \sin \left(\theta_{X}^{\prime}\right) J_{\mathrm{EM}}^{\mu}\right] .
\end{aligned}
$$

Therefore, we find that dark-neutral gauge bosons, $\tilde{Z}^{\prime}$ and $\tilde{X}_{3}$, couple to electromagnetic currents and dark-neutral currents as well as $Z^{\prime}$ currents. Since $\tilde{X}_{3}$ has mass comparable to dark matter mass by the approximate custodial symmetry in the dark sector, it can play an important role for the kinetic equilibrium of dark matter by elastic scattering.

As a result, $\tilde{Z}^{\prime}$ can decay into either a lepton pair or a pair of dark matter particles. Then, the total decay width of $\tilde{Z}^{\prime}$ is given by

$$
\Gamma_{\tilde{Z}^{\prime}}=\Gamma_{\tilde{Z}^{\prime} \rightarrow f \bar{f}}+\Gamma_{\tilde{Z}^{\prime} \rightarrow X^{+} X^{-}}
$$


with

$$
\begin{aligned}
\Gamma_{\tilde{Z}^{\prime} \rightarrow f \bar{f}}= & \frac{N_{c} Q_{f}^{2} \epsilon^{2} \cos ^{2}\left(\theta_{X}^{\prime}\right)\left(2 m_{f}^{2}+m_{\tilde{Z}^{\prime}}^{2}\right)}{12 \pi m_{\tilde{Z}^{\prime}}} \sqrt{1-\frac{4 m_{f}^{2}}{m_{\tilde{Z}^{\prime}}^{2}}} \\
\Gamma_{\tilde{Z}^{\prime} \rightarrow X^{+} X^{-}}= & \frac{g_{X}^{2} \sin ^{2}\left(\theta_{X}^{\prime}\right) m_{\tilde{Z}^{\prime}}}{192 \pi}\left(\frac{m_{\tilde{Z}^{\prime}}}{m_{X}}\right)^{4} \sqrt{1-\frac{4 m_{X}^{2}}{m_{\tilde{Z}^{\prime}}^{2}}}\left[1+16\left(\frac{m_{X}}{m_{\tilde{Z}^{\prime}}}\right)^{2}\right. \\
& \left.-68\left(\frac{m_{X}}{m_{\tilde{Z}^{\prime}}}\right)^{4}-48\left(\frac{m_{X}}{m_{\tilde{Z}^{\prime}}}\right)^{6}\right]
\end{aligned}
$$

On the other hand, $\tilde{X}_{3}$ has mass $m_{\tilde{X}_{3}}<2 m_{X}$ for SIMP processes to be kinematically open. So, $\tilde{X}_{3}$ decays dominantly into a pair of leptons with $f$ being electron or muon, with the decay rate given by

$$
\Gamma_{\tilde{X}_{3} \rightarrow f \bar{f}}=\frac{e^{2} \epsilon^{2} \sin ^{2}\left(\theta_{X}^{\prime}\right)\left(2 m_{f}^{2}+m_{\tilde{X}_{3}}^{2}\right)}{12 \pi m_{\tilde{X}_{3}}} \sqrt{1-\frac{4 m_{f}^{2}}{m_{\tilde{X}_{3}}^{2}}} .
$$

For light dark-neutral gauge bosons of mass around QCD scale, in particular, $\tilde{X}_{3}$, whose mass is close to DM mass by custodial symmetry, we also need to include the decay modes into hadronic states. The hadronic width is given by

$$
\Gamma_{\tilde{X}_{3} \rightarrow \text { hadrons }}=\frac{e^{2} \epsilon^{2} \sin ^{2}\left(\theta_{X}^{\prime}\right) m_{\tilde{X}_{3}}}{12 \pi} \cdot R\left(m_{\tilde{X}_{3}}^{2}\right)
$$

where $R(s)$ is the ratio of hadronic cross section to muonic cross section at tree level [78] in $e^{+} e^{-}$annihilations at center of mass energy $\sqrt{s}$,

$$
R(s) \equiv \frac{\sigma\left(e^{+} e^{-} \rightarrow \text { hadron }\right)}{\sigma\left(e^{+} e^{-} \rightarrow \mu^{+} \mu^{-}\right)} .
$$

When $\tilde{Z}^{\prime}$ is light, we need to include the hadronic decays for $\tilde{Z}^{\prime}$ instead of quark decays by

$$
\Gamma_{\tilde{Z}^{\prime} \rightarrow \text { hadrons }}=\frac{e^{2} \epsilon^{2} \cos ^{2}\left(\theta_{X}^{\prime}\right) m_{\tilde{Z}^{\prime}}}{12 \pi} \cdot R\left(m_{\tilde{Z}^{\prime}}^{2}\right) .
$$

The ratio $R(s)$ can be significantly greater than unity for $600 \mathrm{MeV} \lesssim m_{\tilde{X}_{3}}, m_{\tilde{Z}^{\prime}} \lesssim 2 \mathrm{GeV}$ near hadronic resonances such as $\rho, \omega, \phi, \rho^{\prime}$, etc [78]. Apart from those resonance regions, we can still apply the limits from visible modes with dileptons in our later discussion. But, we note that care should be taken of in interpreting our results for visible modes of light dark-neutral gauge bosons near hadronic resonances. On the other hand, the limits from invisible decays in the later section will be robust in most of parameter space of our interest, because they will dominate once open.

On left of figure 8 , we depicted contours of the ratio of visible to invisible decay rates of $\tilde{Z}^{\prime}$ gauge boson in the parameter space for $\varepsilon$ vs $\alpha_{X} \equiv g_{X}^{2} /(4 \pi)$. Here, we have fixed $m_{X}=100 \mathrm{MeV}, m_{\tilde{Z}^{\prime}}=1 \mathrm{GeV}$ and $\sin \left(2 \theta_{X}^{\prime}\right)=0.1$. So, in most of the parameter space for $\alpha_{X} \gtrsim 0.1$ and $\varepsilon \lesssim 10^{-2}$, the visible decay of $\tilde{Z}^{\prime}$ is negligibly small. On right of figure 8 , we 

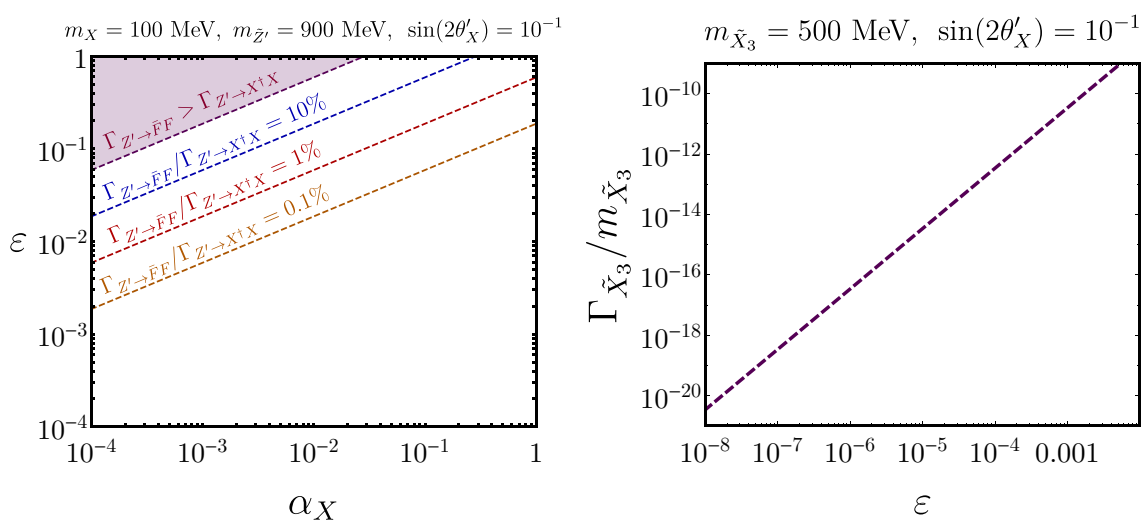

Figure 8. (Left) Ratio of visible to invisible decay rates of $\tilde{Z}^{\prime}$ in the parameter space for $\varepsilon$ and $\alpha_{X}$. (Right) Ratio of decay rate to mass of $\tilde{X}_{3}$ as a function of $\varepsilon$.

also showed the ratio of decay rate to mass of $\tilde{X}_{3}$ as a function of $\varepsilon$, for $m_{\tilde{X}_{3}}=500 \mathrm{MeV}$ and $\sin \left(2 \theta_{X}^{\prime}\right)=0.1$. In this case, for $m_{X}<m_{\tilde{X}_{3}}<2 m_{X}, m_{\tilde{X}_{3}}$ decays visibly into a lepton pair, so the decay rate is doubly suppressed by the dark Weinberg angle and the gauge kinetic parameter.

\subsection{Kinetic equilibrium}

We consider the elastic scattering between dark matter and electron through $Z^{\prime}$ portal couplings to achieve a kinetic equilibrium for SIMP dark matter. In this case, the corresponding momentum relaxation rate during freeze-out is given by

$$
\gamma(T) \simeq \frac{31 \pi^{3} e^{2} \epsilon^{2} g_{X}^{2} \sin ^{2}\left(2 \theta_{X}^{\prime}\right) T^{6}}{1512 m_{X}}\left(\frac{1}{m_{\tilde{X}_{3}}^{2}}-\frac{1}{m_{\tilde{Z}^{\prime}}^{2}}\right)^{2} .
$$

Them, for kinetic equilibrium during SIMP freeze-out, we need to require

$$
\gamma\left(T_{\mathrm{F}}\right)>H\left(T_{\mathrm{F}}\right)\left(\frac{m_{X}}{T_{\mathrm{F}}}\right)^{2}
$$

where $T_{\mathrm{F}} \simeq m_{X} / 15$ is the freeze-out temperature and $H\left(T_{\mathrm{F}}\right)$ is the Hubble parameter at freeze-out.

On the other hand, since dark-neutral gauge boson $\tilde{X}_{3}$ has a similar mass as dark matter in our model, its abundance is not that suppressed around freeze-out temperature. Thus, $\tilde{X}_{3}$ plays an important role of keeping dark matter in thermal equilibrium through the SM by the decays into electron or muon pairs, the kinetic equilibrium for dark matter can be also achieved by the elastic scattering between dark matter and $\tilde{X}_{3}$. Thus, in this case, we require

$$
n_{\tilde{X}_{3}}^{\mathrm{eq}} \Gamma_{\tilde{X}_{3}}>H\left(T_{f}\right) n_{X}^{\mathrm{eq}}
$$

We find that kinetic equilibrium for dark matter can be easily achieved by the elastic scattering between dark matter and dark-neutral gauge boson $\tilde{X}_{3}$ as far as the latter remains in kinetic equilibrium for a tiny gauge kinetic mixing $\epsilon \sim 10^{-6}$ in the parameter space of our interest. 

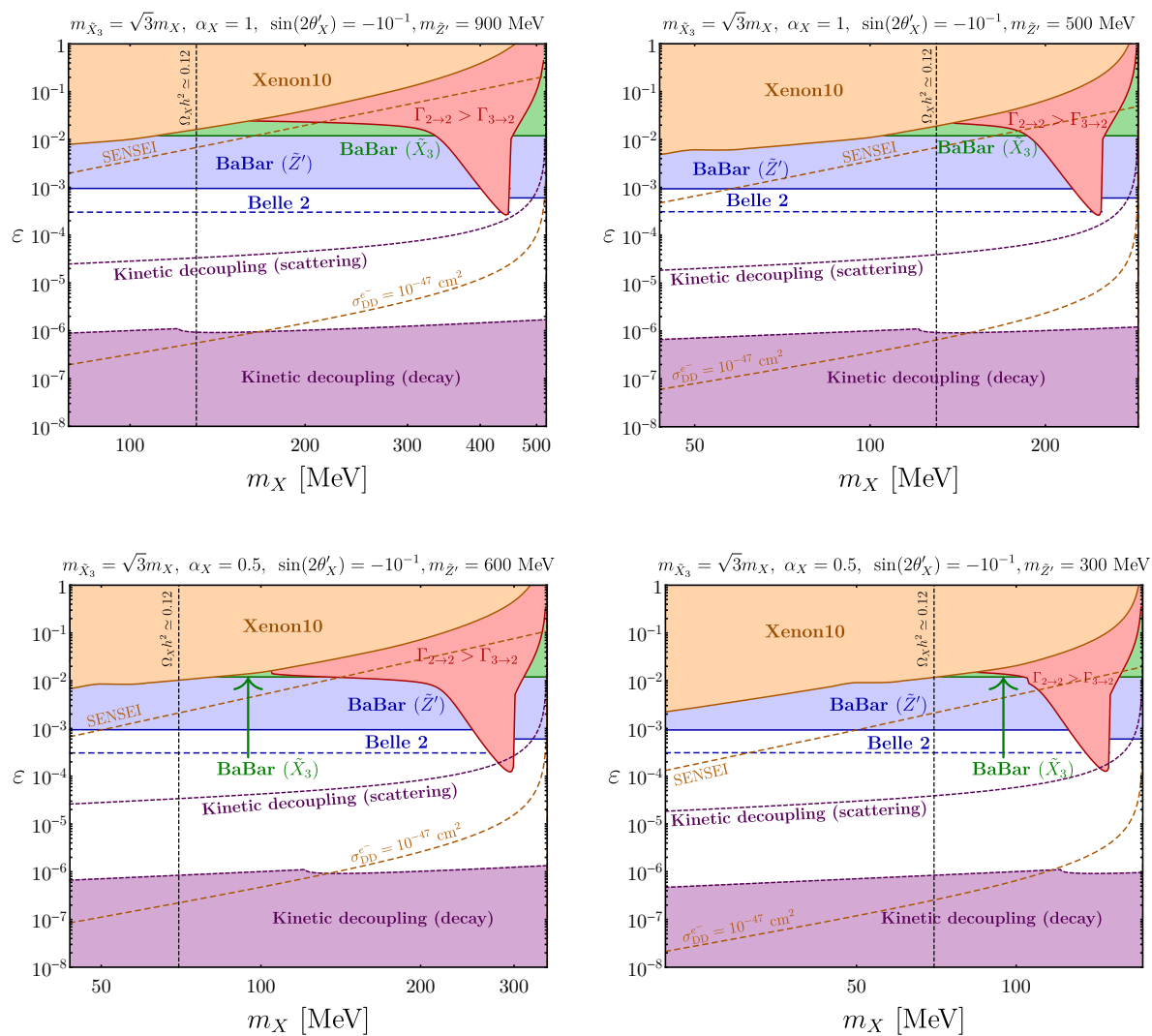

Figure 9. Various constraints on $\varepsilon$ vs $m_{X}$ for the case with quadruplet dark Higgs. The blue region and the region above the blue dashed line are excluded by invisible modes of $\tilde{Z}^{\prime}$ in BaBar [79] (observed) or Belle2 [80] (expected), respectively. The green region is excluded by visible modes of $\tilde{X}_{3}$ in BaBar [82] (dilepton+monophoton: observed). Direct detection limit on DM-electron elastic scattering from XENON10 [83-85] (observed) and SENSEI-100 1yr [86, 87] (expected) are shown in yellowish region and red dashed line, respectively, and contours with DM-electron elastic scattering cross section, $\sigma_{\mathrm{DD}}^{e^{-}}=10^{-47} \mathrm{~cm}^{2}$, are also shown in orange dashed lines. Kinetic decoupling occurs in purple region and $2 \rightarrow 2$ visible annihilations would be dominant in red region. For comparison, the DM-electron scattering process becomes negligible for kinetic equilibrium in the region below the purple dashed line. The relic density is saturated along the dashed black vertical lines.

In figure 9 , we showed the parameter space for $\varepsilon \approx c_{W} \xi$ vs $m_{X}$ with various constraints coming from the model consistency and experiments. We have assumed that the VEV of a quadruplet dark Higgs determines $\mathrm{SU}(2)_{X}$ gauge boson masses and chosen different values for the DM self-coupling $\alpha_{X}=1,0.5$ in the top and bottom panels, respectively, varying $m_{\tilde{Z}^{\prime}}$. We have similar plots for $\varepsilon$ vs $m_{\tilde{Z}^{\prime}}$ in figure 10 .

As for the theoretical constraints, dark matter becomes out of kinetic equilibrium already during freeze-out in purple region and $2 \rightarrow 2$ visible annihilations such as $X_{+} X_{-} \rightarrow$ $\tilde{X}_{3} \tilde{X}_{3}$ would be dominant in red region. The relic density is saturated along the black dashed vertical line in figure 9, which is determined mostly by the SIMP processes. In figure 10, we have chosen the values of $m_{X}$ and $\alpha_{X}$ such that the correct relic density is saturated. 

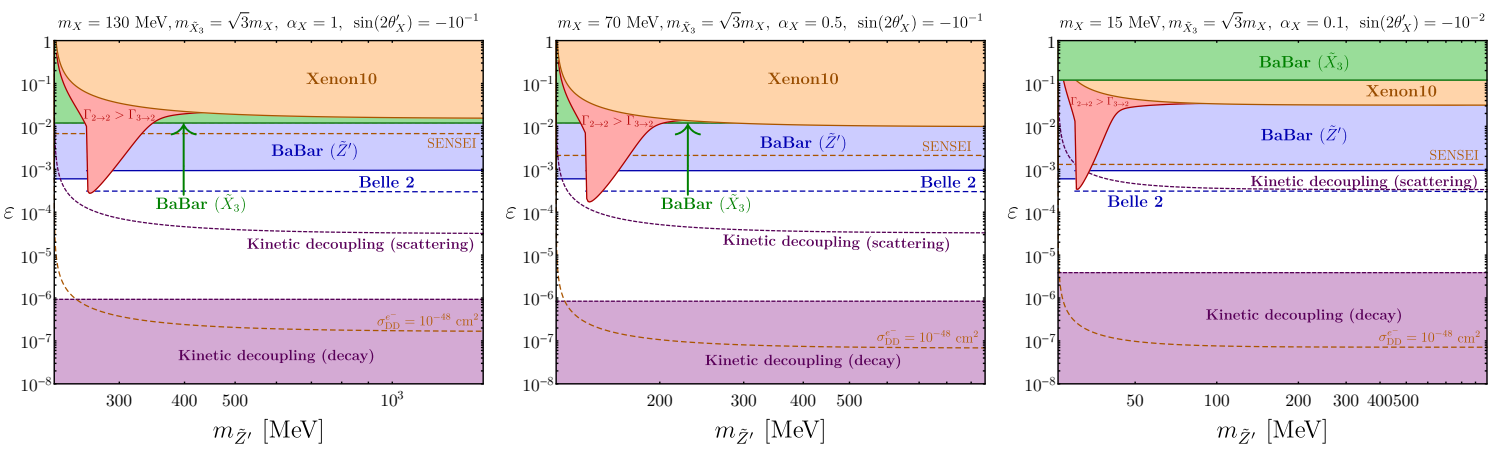

Figure 10. Similar constraints as in figure 9 , but in the parameter space for $\varepsilon$ vs $m_{\tilde{Z}^{\prime}}$ for the case with quadruplet dark Higgs.

We now turn to the experimental constraints on the model. As $\tilde{Z}^{\prime}$ decays mostly invisibly into a dark matter pair, the observed limit from BaBar [79] and the future projection [80] from Belle $2^{3}$ can rule out the parameter space in blue region and in the region above the blue dashed line. On the other hand, $\tilde{X}_{3}$ decays visibly into a lepton pair in the SM so there are constraints from the monphoton+dilepton searches in BaBar [82], but they are not as strong as the invisible searches for $\tilde{Z}^{\prime}$ in BaBar. Direct detection from electron-recoil signals in XENON10 [83-85] has excluded the yellowish region, sometimes being comparable than or even stronger than the visible bound from BaBar. The future updated SENSEI-100 1yr [86, 87] can reach the limit for light $\tilde{Z}^{\prime}$ mediator, even beyond the Belle2 projection. For comparison, we also showed the contours with DM-electron elastic scattering cross section, $\sigma_{\mathrm{DD}}^{e^{-}}=10^{-47} \mathrm{~cm}^{2}$, in orange dashed lines.

\subsection{SM $2 \rightarrow 2$ annihilations and direct detection}

Light dark matter can also annihilate into a pair of leptons or mesons for $m_{X} \lesssim 1 \mathrm{GeV}$. In particular, the annihilation cross section for $X_{+} X_{-} \rightarrow f \bar{f}$, with $f$ being electron or muon, is before thermal average,

$$
\left(\sigma v_{\mathrm{rel}}\right)_{X_{+} X_{-} \rightarrow f \bar{f}}=\frac{e^{2} \epsilon^{2} g_{X}^{2} \sin ^{2}\left(2 \theta_{X}^{\prime}\right)\left(m_{f}^{2}+2 m_{X}^{2}\right)\left(m_{\tilde{X}_{3}}^{2}-m_{\tilde{Z}^{\prime}}^{2}\right)^{2}}{16 \pi\left(4 m_{X}^{2}-m_{\tilde{X}_{3}}^{2}\right)^{2}\left(4 m_{X}^{2}-m_{\tilde{Z}^{\prime}}^{2}\right)^{2}} \sqrt{1-\frac{m_{f}^{2}}{m_{X}^{2}}} v_{\mathrm{rel}}^{2} .
$$

The thermal average of the above annihilation cross section needs care near the resonances, except which, the DM $2 \rightarrow 2$ annihilations are velocity-suppressed so they are not constrained by indirect detection experiments [50].

The $\tilde{X}_{3}$ and $\tilde{Z}^{\prime}$ couple to both dark matter and electron so direct detection for light dark matter is relevant. For $m_{e}, m_{X}, m_{\tilde{Z}^{\prime}}, m_{\tilde{X}_{3}} \gg p_{X} \simeq m_{X} v_{\mathrm{X}}$, the DM-electron elastic scattering cross section is approximately given by

$$
\begin{aligned}
\sigma_{\mathrm{DD}}^{e^{-}} & =\frac{e^{2} \epsilon^{2} g_{X}^{2} \sin ^{2}\left(2 \theta_{X}^{\prime}\right) m_{e}^{2} m_{X}^{2}}{4 \pi\left(m_{e}+m_{X}\right)^{2}}\left(\frac{1}{m_{\tilde{X}_{3}}^{2}}-\frac{1}{m_{\tilde{Z}^{\prime}}^{2}}\right)^{2} \\
& \approx 9.3 \times 10^{-42} \mathrm{~cm}^{2}\left(\frac{\varepsilon}{10^{-4}}\right)^{2}\left(\frac{\alpha_{X}}{1}\right)\left(\frac{\sin \left(2 \theta_{X}^{\prime}\right)}{0.1}\right)^{2}\left(\frac{100 \mathrm{MeV}}{m_{\tilde{X}_{3}}}\right)^{2}\left(1-\frac{m_{\tilde{X}_{3}}^{2}}{m_{\tilde{Z}^{\prime}}^{2}}\right)^{2} .
\end{aligned}
$$

\footnotetext{
${ }^{3}$ See also ref. [81] for the previous estimates on Belle2 sensitivity.
} 
Thus, there are two mediators contributing to the DM $2 \rightarrow 2$ annihilation and the DMelectron elastic scattering in our case, in particular, we can impose the direct detection constraints on the $Z^{\prime}$ portal coupling with a single mediator by identifying $1 / m_{Z^{\prime}}^{4}$ with $\left(1 / m_{\tilde{X}_{3}}^{2}-1 / m_{\tilde{Z}^{\prime}}^{2}\right)^{2}$ as we have discussed for figures 9 and 10 .

\section{Conclusions}

We have proposed a new model for Vector SIMP dark matter in the context of dark $\mathrm{SU}(2)_{X} \times \mathrm{U}(1)_{Z^{\prime}}$ gauge theory with a similarity to the SM counterpart. The mass splitting between dark matter and neutral components of $\mathrm{SU}(2)_{X}$ are predicted by an approximate custodial symmetry in the dark Higgs sector, playing a crucial role for the production mechanism of dark matter due to self-interactions. The kinetic equilibrium for VSIMP is maintained during the freeze-out thanks to a gauge kinetic mixing between $\mathrm{U}(1)_{Z^{\prime}}$ and the $\mathrm{SM}$, providing a testing ground for searches for light mediators of order $\mathrm{GeV}$ scale or below at current and future collider and direct detection experiments.

\section{Acknowledgments}

The work of YM was also supported by the France-US PICS MicroDark. The work of HML and SMC is supported in part by Basic Science Research Program through the National Research Foundation of Korea (NRF) funded by the Ministry of Education, Science and Technology (NRF-2016R1A2B4008759, NRF-2018R1A4A1025334 and NRF2019R1A2C2003738). The work of SMC is supported in part by TJ Park Science Fellowship of POSCO TJ Park Foundation. The work of MP was supported by the Spanish Agencia Estatal de Investigación through the grants FPA2015-65929-P (MINECO/FEDER, UE), IFT Centro de Excelencia Severo Ochoa SEV-2016-0597, and Red Consolider MultiDark FPA2017-90566-REDC. This research has also been supported by the (Indo-French) CEFIPRA/IFCPAR Project No. 5404-2. Support from CNRS LIA-THEP and the INFREHEPNET of CEFIPRA/IFCPAR is also acknowledged. YM acknowledges partial support from the European Union Horizon 2020 research and innovation programme under the Marie Sklodowska-Curie: RISE InvisiblesPlus (grant agreement No 690575) and the ITN Elusives (grant agreement No 674896).

\section{A General dark gauge boson masses}

We discuss general gauge boson masses in the dark sector in our model. In the presence of a dark Higgs field $F$ in the representation with $\mathrm{SU}(2)_{X}$ isospin $I$, general dark gauge boson mass terms are given [88] by

$$
\begin{aligned}
\mathcal{L}_{\mathrm{SSB}}= & F^{\dagger}\left(g_{X}^{2}\left[I(I+1)-\left(I_{3}\right)^{2}\right] X_{\mu}^{\dagger} X^{\mu}+g_{X}^{2}\left(I_{3}\right)^{2} X_{3 \mu} X_{3}^{\mu}\right. \\
& \left.+g_{Z^{\prime}}^{2}\left(Z^{\prime}\right)^{2} Z_{\mu}^{\prime} Z^{\prime \mu}+2 g_{X} g_{Z^{\prime}}\left(Z^{\prime} I_{3}\right) Z_{\mu}^{\prime} X_{3}^{\mu}\right) F
\end{aligned}
$$

For a singlet $S$, a doublet $\Phi$, a triplet $T$, a quadruplet $Q_{4}$, a quintuplet $Q_{5}$, etc, with $I=\frac{1}{2}, 1, \frac{3}{2}, 2$, etc, respectively, we assign $Z^{\prime}=q_{S}$ and $Z^{\prime}=I_{3}=\frac{1}{2}, 1, \frac{3}{2}, 2$, etc, resulting 
in $I(I+1)-\left(I_{3}\right)^{2}=I=\frac{1}{2}, 1, \frac{3}{2}, 2$, etc. Then, for nonzero VEVs with $S=\frac{1}{\sqrt{2}} v_{S}$, $\langle\Phi\rangle=\frac{1}{\sqrt{2}}\left(0, v_{\Phi}\right)^{T}$, and $T=\left(0,0, \frac{1}{\sqrt{2}} v_{T}\right)^{T}, Q_{4}=\left(0,0,0, \frac{1}{\sqrt{2}} v_{Q_{4}}\right)^{T}, Q_{5}=\left(0,0,0,0, \frac{1}{\sqrt{2}} v_{Q_{5}}\right)^{T}$, etc, the dark-charged gauge bosons, $X_{\mu}, X_{\mu}^{\dagger}$, have masses,

$$
m_{X}^{2}=\frac{1}{2} g_{X}^{2} \sum_{I} I v_{I}^{2}
$$

where $v_{I}=v_{\Phi}, v_{T}, v_{Q_{4}}, v_{Q_{5}}$, etc. On the other hand, the mass matrix for neutral gauge bosons, $Z_{\mu}^{\prime}$ and $X_{3 \mu}$, takes the following form,

$$
M_{2 \times 2}^{2}=m_{X_{3}}^{2}\left(\begin{array}{cc}
\beta s_{X}^{2} & -s_{X} c_{X} \\
-s_{X} c_{X} & c_{X}^{2}
\end{array}\right)
$$

where $m_{X_{3}}^{2} \equiv\left(g_{X}^{2}+g_{Z^{\prime}}^{2}\right) \sum_{I} I^{2} v_{I}^{2}, c_{X} \equiv \cos \theta_{X}$ and $s_{X} \equiv \sin \theta_{X}$, with $\sin \theta_{X}=g_{Z^{\prime}} / \sqrt{g_{X}^{2}+g_{Z^{\prime}}^{2}}$, and

$$
\beta \equiv 1+\frac{q_{S}^{2} v_{S}^{2}}{\sum_{I} I^{2} v_{I}^{2}} .
$$

In the absence of the gauge kinetic mixing, the above mass matrix (A.3) can be diagonalized by introducing a dark Weinberg angle as in the SM. Performing a rotation of dark gauge fields to mass eigenstates, $\tilde{Z}_{\mu}^{\prime}, \tilde{X}_{3 \mu}$, as

$$
\left(\begin{array}{c}
Z_{\mu}^{\prime} \\
X_{3 \mu}
\end{array}\right)=\left(\begin{array}{cc}
\cos \theta_{X}^{\prime} & -\sin \theta_{X}^{\prime} \\
\sin \theta_{X}^{\prime} & \cos \theta_{X}^{\prime}
\end{array}\right)\left(\begin{array}{c}
\tilde{Z}_{\mu}^{\prime} \\
\tilde{X}_{3 \mu}
\end{array}\right)
$$

with

$$
\tan \left(2 \theta_{X}^{\prime}\right)=\frac{2 c_{X} s_{X}}{c_{X}^{2}-\beta s_{X}^{2}}
$$

we obtain the mass eigenvalues for dark gauge bosons,

$$
\begin{aligned}
& m_{\tilde{Z}^{\prime}}^{2}=m_{X_{3}}^{2} c_{X}^{2}\left(1-\cot \theta_{X}^{\prime} \tan \theta_{X}\right), \\
& m_{\tilde{X}_{3}}^{2}=m_{X_{3}}^{2} c_{X}^{2}\left(1+\tan \theta_{X}^{\prime} \tan \theta_{X}\right) .
\end{aligned}
$$

The results generalize our results in eqs. (3.1)-(3.9) with a singlet $S$ and a triplet $T$ in the main text.

For $\beta \gg 1$, from eq. (A.6), we get $\tan \theta_{X}^{\prime} \approx-\frac{1}{\beta \tan \theta_{X}}$, leading to the approximate gauge boson masses,

$$
\begin{aligned}
& m_{\tilde{Z}^{\prime}}^{2} \approx g_{X}^{2}\left(\sum_{I} I^{2} v_{I}^{2}\right)\left(1+\beta \tan ^{2} \theta_{X}\right), \\
& m_{\tilde{X}_{3}}^{2} \approx g_{X}^{2}\left(\sum_{I} I^{2} v_{I}^{2}\right)\left(1-\frac{1}{\beta}\right) .
\end{aligned}
$$

In this limit, the mass difference between the light gauge bosons is given by

$$
m_{\tilde{X}_{3}}^{2}-m_{X}^{2} \approx g_{X}^{2} \sum_{I} I\left(I-\frac{1}{2}\right) v_{I}^{2}-\frac{1}{\beta} g_{X}^{2} \sum_{I} I^{2} v_{I}^{2} .
$$


Therefore, for $m_{\tilde{X}_{3}}^{2}>m_{X}^{2}$, we need $I>\frac{1}{2}$, namely, at least a triplet dark Higgs with nonzero VEV.

For instance, ignoring the mass splitting due to the dark Weinberg mixing and keeping only one Higgs representation with $I=\frac{1}{2}, 1, \frac{3}{2}, 2$, we get

$$
m_{\tilde{X}_{3}}^{2} \approx m_{X}^{2}, \quad 2 m_{X}^{2}, \quad 3 m_{X}^{2}, \quad 4 m_{X}^{2}
$$

Then, we get $\Delta \equiv\left(m_{\tilde{X}_{3}}-m_{X}\right) / m_{X}$ for $I=\frac{1}{2}, 1, \frac{3}{2}, 2$, as follows,

$$
\Delta=0, \quad \sqrt{2}-1, \quad \sqrt{3}-1, \quad 1 .
$$

But, for general VEVs of all Higgs representations with $\frac{1}{2} \leq I \leq 2$, we can cover the entire range of the mass splitting continuously for $0 \leq \Delta \leq 2$.

\section{B Dark Higgs masses}

In this appendix, we discuss general dark-charged and neutral Higgs boson masses in the presence of dark gauge symmetry breaking in our model and ensure that extra Higgs bosons can be safely decoupled in our consideration.

Triplet Higgs bosons. Minimizing the scalar potential for the dark triplet Higgs in eq. (2.2), we find that the VEV of the dark triplet Higgs is related to the parameters in the scalar potential as follows,

$$
v_{T}=\sqrt{\frac{m_{T}^{2}}{\lambda_{T}-\tilde{\lambda}_{T}}} .
$$

Then, together with the Higgs portal coupling in eq. (2.5), dark Higgs bosons in the triplet receive masses,

$$
m_{h_{T}}^{2}=2 v_{T}^{2}\left(\lambda_{T}-\tilde{\lambda}_{T}\right)-\frac{1}{2} \lambda_{T H} v^{2}, \quad m_{h^{++}}^{2}=2 v_{T}^{2} \tilde{\lambda}_{T}-\frac{1}{2} \lambda_{T H} v^{2},
$$

and there is a mixing between the neutral dark Higgs $h_{T}$ and the SM Higgs $h$ by

$$
\left(\begin{array}{l}
h_{1} \\
h_{2}
\end{array}\right)=\left(\begin{array}{cc}
\cos \theta & -\sin \theta \\
\sin \theta & \cos \theta
\end{array}\right)\left(\begin{array}{c}
h_{T} \\
h
\end{array}\right)
$$

where $h_{1,2}$ are mass eigenstates: $h_{1}$ is the triplet-like Higgs and $h_{2}$ the SM-doublet like Higgs. Then, the mass eigenvalues of neutral scalars are

$$
\begin{aligned}
m_{h_{1,2}}^{2}= & \frac{1}{4} v^{2}\left(4 \lambda_{H}-\lambda_{T H}\right)-\frac{1}{4} v_{T}^{2}\left(4\left(\tilde{\lambda}_{T}-\lambda_{T}\right)+\lambda_{T H}\right) \\
\mp & \frac{1}{4}\left[\left(v^{2}\left(4 \lambda_{H}-\lambda_{T H}\right)-v_{T}^{2}\left(4\left(\tilde{\lambda}_{T}-\lambda_{T}\right)+\lambda_{T H}\right)\right)^{2}\right. \\
& \left.+4 v_{T}^{2} \lambda_{T H}\left(3 v^{2} \lambda_{T H}-4 v_{T}^{2}\left(\tilde{\lambda}_{T}-\lambda_{T}\right)\right)+16 v^{2} \lambda_{H}\left(v^{2} \lambda_{T H}+4 v_{T}^{2}\left(\tilde{\lambda}_{T}-\lambda_{T}\right)\right)\right]^{1 / 2}
\end{aligned}
$$


and the mixing angle is

$$
\tan 2 \theta=-\frac{4 v v_{T} \lambda_{T H}}{\lambda_{T H} v^{2}+4 \lambda_{H} v^{2}-v_{T}^{2}\left(-4 \tilde{\lambda}_{T}+4 \lambda_{T}+\lambda_{T H}\right)} .
$$

In the limit of a small $\lambda_{T H}$, the mass eigenvalues of neutral scalars become

$$
m_{h_{1}}^{2} \approx 2 v_{T}^{2}\left(\lambda_{T}-\tilde{\lambda}_{T}\right), \quad m_{h_{2}}^{2} \approx 2 \lambda_{H} v^{2}
$$

Then, the vacuum stability bound, $\lambda_{T}>\tilde{\lambda}_{T}$, ensures the positive squared masses for neutral dark Higgs boson, $h_{1} \approx h_{T}$, for a small mixing quartic coupling.

In order to ensure the consistency of the dark vacuum, we require $m_{h^{++}}^{2}>0$ or

$$
\lambda_{T H}<\frac{4 v_{T}^{2} \tilde{\lambda}_{T}}{v^{2}}
$$

which naturally pushes the value of the quartic mixing to be $\lambda_{T H} \lesssim 10^{-5}$ for $v_{T} \sim \mathrm{GeV}$, implying the mixing angle $\tan 2 \theta \lesssim 10^{-5}$ to be below the bound from the Higgs invisible decay.

Moreover, from the kinetic terms of the triplet complex field in eq. (2.2), we also derive the following interactions between dark Higgs and gauge bosons,

$$
\begin{aligned}
\mathcal{L}_{\mathrm{DH}-\mathrm{DG}}= & i h^{++} \stackrel{\leftrightarrow}{\partial}_{\mu}\left(h^{++}\right)^{\dagger}\left(g_{X} X_{3}^{\mu}+g_{Z^{\prime}} Z^{\prime \mu}\right)+g_{X}^{2}\left(\frac{v_{T}+h_{T}}{\sqrt{2}}\right)\left(\left(h^{++}\right)^{\dagger} X^{\mu} X_{\mu}+h^{++} X_{\mu}^{\dagger} X^{\dagger \mu}\right) \\
& +\left|h^{++}\right|^{2}\left(g_{Z^{\prime}}^{2} Z^{\prime \mu} Z_{\mu}^{\prime}+2 g_{X} g_{Z^{\prime}} Z^{\prime \mu} X_{3 \mu}+g_{X}^{2}\left(X_{3}^{\mu} X_{3 \mu}+X^{\dagger \mu} X_{\mu}\right)\right) \\
& +\left(\frac{1}{2} h_{T}^{2}+v_{T} h_{T}\right)\left(g_{Z^{\prime}}^{2} Z^{\prime \mu} Z_{\mu}^{\prime}-2 g_{X} g_{Z^{\prime}} Z^{\prime \mu} X_{3 \mu}+g_{X}^{2}\left(X_{3}^{\mu} X_{3 \mu}+X^{\dagger \mu} X_{\mu}\right)\right) .
\end{aligned}
$$

Quadruplet Higgs bosons. For the quadruplet Higgs $Q_{4}=\left(h^{(3)}, h^{(2)}, 0, \frac{1}{\sqrt{2}}\left(v_{Q_{4}}+h_{Q_{4}}\right)\right)^{T}$ with

$$
v_{Q_{4}}=\frac{2 m_{Q_{4}}}{\sqrt{4 \lambda_{Q_{4}}-9 \tilde{\lambda}_{Q_{4}}}}
$$

the masses of dark-charged and neutral Higgs bosons are

$$
\begin{aligned}
& m_{h^{(2)}}^{2}=3 \tilde{\lambda}_{Q_{4}} v_{Q_{4}}^{2}-\frac{1}{2} \lambda_{Q_{4} H} v^{2} \\
& m_{h^{(3)}}^{2}=\frac{9}{2} \tilde{\lambda}_{Q_{4}} v_{Q_{4}}^{2}-\frac{1}{2} \lambda_{Q_{4} H} v^{2}, \\
& m_{h_{Q_{4}}}^{2}=\left(2 \lambda_{Q_{4}}-\frac{9}{2} \tilde{\lambda}_{Q_{4}}\right) v_{Q_{4}}^{2}-\frac{1}{2} \lambda_{Q_{4} H} v^{2} .
\end{aligned}
$$

As a result, there are similar consistent conditions on the mixing quartic couplings for $m_{h^{(2)}}^{2}>0$ and $m_{h^{(2)}}^{3}>0$, as in eq. (B.7). We can ignore the dark charged Higgs contributions in the later discussion, if they are heavy enough for $\lambda_{Q_{4}} v_{Q_{4}}^{2} \sim \tilde{\lambda}_{Q_{4}} v_{Q_{4}}^{2} \gg m_{X}^{2}$. Moerover, a similar vacuum stability bound, $\lambda_{Q_{4}}>\frac{9}{4} \tilde{\lambda}_{Q_{4}}$, ensures the positive squared masses for neutral dark Higgs boson $h_{Q_{4}}$, for a small mixing quartic coupling. 
Quintuplet Higgs bosons. For the quintuplet Higgs $Q_{5}=\left(h^{(4)}, h^{(3)}, h^{(2)}, 0, \frac{1}{\sqrt{2}}\left(v_{Q_{5}}+h_{Q_{5}}\right)\right)^{T}$ with

$$
v_{Q_{5}}=\frac{m_{Q_{5}}}{\sqrt{\lambda_{Q_{5}}-4 \tilde{\lambda}_{Q_{5}}}},
$$

the masses of dark-charged and neutral Higgs are

$$
\begin{aligned}
& m_{h^{(2)}}^{2}=4 \tilde{\lambda}_{Q_{5}} v_{Q_{5}}^{2}-\frac{1}{2} \lambda_{Q_{5} H} v^{2}, \\
& m_{h^{(3)}}^{2}=6 \tilde{\lambda}_{Q_{5}} v_{Q_{5}}^{2}-\frac{1}{2} \lambda_{Q_{5} H} v^{2} \\
& m_{h^{(4)}}^{2}=8 \tilde{\lambda}_{Q_{5}} v_{Q_{5}}^{2}-\frac{1}{2} \lambda_{Q_{5} H} v^{2} \\
& m_{h_{Q_{5}}}^{2}=2\left(\lambda_{Q_{5}}-4 \tilde{\lambda}_{Q_{5}}\right) v_{Q_{5}}^{2}-\frac{1}{2} \lambda_{Q_{5} H} v^{2} .
\end{aligned}
$$

As a result, there are similar consistent conditions on the mixing quartic couplings for $m_{h^{(a)}}^{2}>0$ with $a=1,2,3$, as in eq. (B.7). Similarly, he dark charged Higgs contributions can be neglected in the later discussion, when $\lambda_{Q_{5}} v_{Q_{5}}^{2} \sim \tilde{\lambda}_{Q_{5}} v_{Q_{5}}^{2} \gg m_{X}^{2}$. Moerover, a similar vacuum stability bound, $\lambda_{Q_{5}}>4 \tilde{\lambda}_{Q_{5}}$, ensures the positive squared masses for neutral dark Higgs boson $h_{Q_{5}}$, for a small mixing quartic coupling.

Open Access. This article is distributed under the terms of the Creative Commons Attribution License (CC-BY 4.0), which permits any use, distribution and reproduction in any medium, provided the original author(s) and source are credited.

\section{References}

[1] Planck collaboration, Planck 2015 results. XIII. Cosmological parameters, Astron. Astrophys. 594 (2016) A13 [arXiv:1502.01589] [INSPIRE].

[2] PlanCK collaboration, Planck 2018 results. VI. Cosmological parameters, arXiv: 1807.06209 [INSPIRE].

[3] LUX collaboration, Results from a search for dark matter in the complete LUX exposure, Phys. Rev. Lett. 118 (2017) 021303 [arXiv: 1608.07648] [INSPIRE].

[4] PandaX-II collaboration, Dark Matter Results From 54-Ton-Day Exposure of PandaX-II Experiment, Phys. Rev. Lett. 119 (2017) 181302 [arXiv:1708.06917] [InSPIRE].

[5] XENON collaboration, First Dark Matter Search Results from the XENON1T Experiment, Phys. Rev. Lett. 119 (2017) 181301 [arXiv:1705.06655] [INSPIRE].

[6] V. Silveira and A. Zee, Scalar phantoms, Phys. Lett. 161B (1985) 136 [InSPIRE].

[7] J. McDonald, Gauge singlet scalars as cold dark matter, Phys. Rev. D 50 (1994) 3637 [hep-ph/0702143] [INSPIRE].

[8] C.P. Burgess, M. Pospelov and T. ter Veldhuis, The Minimal model of nonbaryonic dark matter: A Singlet scalar, Nucl. Phys. B 619 (2001) 709 [hep-ph/0011335] [INSPIRE].

[9] H. Davoudiasl, R. Kitano, T. Li and H. Murayama, The New minimal standard model, Phys. Lett. B 609 (2005) 117 [hep-ph/0405097] [INSPIRE]. 
[10] H. Han and S. Zheng, New Constraints on Higgs-portal Scalar Dark Matter, JHEP 12 (2015) 044 [arXiv: 1509.01765] [INSPIRE].

[11] G. Arcadi, C. Gross, O. Lebedev, S. Pokorski and T. Toma, Evading Direct Dark Matter Detection in Higgs Portal Models, Phys. Lett. B 769 (2017) 129 [arXiv:1611.09675] [INSPIRE].

[12] J.A. Casas, D.G. Cerdeño, J.M. Moreno and J. Quilis, Reopening the Higgs portal for single scalar dark matter, JHEP 05 (2017) 036 [arXiv: 1701.08134] [INSPIRE].

[13] A. Djouadi, O. Lebedev, Y. Mambrini and J. Quevillon, Implications of LHC searches for Higgs-portal dark matter, Phys. Lett. B 709 (2012) 65 [arXiv:1112.3299] [InSPIRE].

[14] A. Djouadi, A. Falkowski, Y. Mambrini and J. Quevillon, Direct Detection of Higgs-Portal Dark Matter at the LHC, Eur. Phys. J. C 73 (2013) 2455 [arXiv:1205.3169] [InSPIRE].

[15] O. Lebedev, H.M. Lee and Y. Mambrini, Vector Higgs-portal dark matter and the invisible Higgs, Phys. Lett. B 707 (2012) 570 [arXiv:1111.4482] [InSPIRE].

[16] Y. Mambrini, Higgs searches and singlet scalar dark matter: Combined constraints from XENON 100 and the LHC, Phys. Rev. D 84 (2011) 115017 [arXiv:1108.0671] [InSPIRE].

[17] J.M. Cline, K. Kainulainen, P. Scott and C. Weniger, Update on scalar singlet dark matter, Phys. Rev. D 88 (2013) 055025 [Erratum ibid. D 92 (2015) 039906] [arXiv:1306.4710] [INSPIRE].

[18] S. Baek, P. Ko, W.-I. Park and E. Senaha, Higgs Portal Vector Dark Matter: Revisited, JHEP 05 (2013) 036 [arXiv:1212.2131] [INSPIRE].

[19] G. Arcadi, A. Djouadi and M. Raidal, Dark Matter through the Higgs portal, arXiv: 1903.03616 [INSPIRE].

[20] J. Ellis, A. Fowlie, L. Marzola and M. Raidal, Statistical Analyses of Higgs- and Z-Portal Dark Matter Models, Phys. Rev. D 97 (2018) 115014 [arXiv:1711.09912] [INSPIRE].

[21] G. Arcadi, Y. Mambrini and F. Richard, Z-portal dark matter, JCAP 03 (2015) 018 [arXiv: 1411.2985] [INSPIRE].

[22] J. Kearney, N. Orlofsky and A. Pierce, $Z$ boson mediated dark matter beyond the effective theory, Phys. Rev. D 95 (2017) 035020 [arXiv:1611.05048] [InSPIRE].

[23] M. Escudero, A. Berlin, D. Hooper and M.-X. Lin, Toward (Finally!) Ruling Out Z and Higgs Mediated Dark Matter Models, JCAP 12 (2016) 029 [arXiv:1609.09079] [INSPIRE].

[24] A. Alves, S. Profumo and F.S. Queiroz, The dark $Z^{\prime}$ portal: direct, indirect and collider searches, JHEP 04 (2014) 063 [arXiv: 1312.5281] [INSPIRE].

[25] C. Gross, O. Lebedev and Y. Mambrini, Non-Abelian gauge fields as dark matter, JHEP 08 (2015) 158 [arXiv: 1505.07480] [INSPIRE].

[26] G. Arcadi, Y. Mambrini, M.H.G. Tytgat and B. Zaldivar, Invisible $Z^{\prime}$ and dark matter: LHC vs LUX constraints, JHEP 03 (2014) 134 [arXiv:1401.0221] [INSPIRE].

[27] O. Lebedev and Y. Mambrini, Axial dark matter: The case for an invisible Z', Phys. Lett. B 734 (2014) 350 [arXiv: 1403.4837] [INSPIRE].

[28] G. Arcadi et al., The waning of the WIMP? A review of models, searches and constraints, Eur. Phys. J. C 78 (2018) 203 [arXiv:1703.07364] [InSPIRE]. 
[29] GAMBIT collaboration, Global analyses of Higgs portal singlet dark matter models using GAMBIT, Eur. Phys. J. C 79 (2019) 38 [arXiv: 1808.10465] [INSPIRE].

[30] L.J. Hall, K. Jedamzik, J. March-Russell and S.M. West, Freeze-In Production of FIMP Dark Matter, JHEP 03 (2010) 080 [arXiv:0911.1120] [INSPIRE].

[31] X. Chu, T. Hambye and M.H.G. Tytgat, The Four Basic Ways of Creating Dark Matter Through a Portal, JCAP 05 (2012) 034 [arXiv: 1112.0493] [INSPIRE].

[32] X. Chu, Y. Mambrini, J. Quevillon and B. Zaldivar, Thermal and non-thermal production of dark matter via $Z^{\prime}$-portal(s), JCAP 01 (2014) 034 [arXiv:1306.4677] [INSPIRE].

[33] A. Biswas, D. Borah and A. Dasgupta, UV complete framework of freeze-in massive particle dark matter, Phys. Rev. D 99 (2019) 015033 [arXiv:1805.06903] [InSPIRE].

[34] Y. Mambrini, K.A. Olive, J. Quevillon and B. Zaldivar, Gauge Coupling Unification and Nonequilibrium Thermal Dark Matter, Phys. Rev. Lett. 110 (2013) 241306 [arXiv: 1302.4438] [INSPIRE].

[35] Y. Mambrini, N. Nagata, K.A. Olive, J. Quevillon and J. Zheng, Dark matter and gauge coupling unification in nonsupersymmetric $\mathrm{SO}(10)$ grand unified models, Phys. Rev. D 91 (2015) 095010 [arXiv: 1502.06929] [INSPIRE].

[36] Y. Mambrini, N. Nagata, K.A. Olive and J. Zheng, Vacuum Stability and Radiative Electroweak Symmetry Breaking in an SO(10) Dark Matter Model, Phys. Rev. D 93 (2016) 111703 [arXiv: 1602.05583] [INSPIRE].

[37] G. Bhattacharyya, M. Dutra, Y. Mambrini and M. Pierre, Freezing-in dark matter through a heavy invisible $Z^{\prime}$, Phys. Rev. D 98 (2018) 035038 [arXiv:1806.00016] [INSPIRE].

[38] N. Bernal, M. Dutra, Y. Mambrini, K. Olive, M. Peloso and M. Pierre, Spin-2 Portal Dark Matter, Phys. Rev. D 97 (2018) 115020 [arXiv:1803.01866] [InSPIRE].

[39] K.-Y. Choi and H.M. Lee, Axino abundances in high-scale supersymmetry, Phys. Dark Univ. 22 (2018) 202 [arXiv:1810.00293] [INSPIRE].

[40] S.-M. Choi, Y.-J. Kang, H.M. Lee and K. Yamashita, Unitary inflaton as decaying dark matter, JHEP 05 (2019) 060 [arXiv:1902.03781] [INSPIRE].

[41] K. Benakli, Y. Chen, E. Dudas and Y. Mambrini, Minimal model of gravitino dark matter, Phys. Rev. D 95 (2017) 095002 [arXiv:1701.06574] [INSPIRE].

[42] E. Dudas, Y. Mambrini and K. Olive, Case for an EeV Gravitino, Phys. Rev. Lett. 119 (2017) 051801 [arXiv: 1704.03008] [InSPIRE].

[43] E. Dudas, T. Gherghetta, Y. Mambrini and K.A. Olive, Inflation and High-Scale Supersymmetry with an EeV Gravitino, Phys. Rev. D 96 (2017) 115032 [arXiv:1710.07341] [INSPIRE].

[44] E. Dudas, T. Gherghetta, K. Kaneta, Y. Mambrini and K.A. Olive, Gravitino decay in high scale supersymmetry with $R$-parity violation, Phys. Rev. D 98 (2018) 015030 [arXiv: 1805. 07342] [INSPIRE].

[45] D. Chowdhury, E. Dudas, M. Dutra and Y. Mambrini, Moduli Portal Dark Matter, Phys. Rev. D 99 (2019) 095028 [arXiv: 1811.01947] [InSPIRE].

[46] Y. Hochberg, E. Kuflik, T. Volansky and J.G. Wacker, Mechanism for Thermal Relic Dark Matter of Strongly Interacting Massive Particles, Phys. Rev. Lett. 113 (2014) 171301 [arXiv: 1402.5143] [INSPIRE]. 
[47] Y. Hochberg, E. Kuflik, H. Murayama, T. Volansky and J.G. Wacker, Model for Thermal Relic Dark Matter of Strongly Interacting Massive Particles, Phys. Rev. Lett. 115 (2015) 021301 [arXiv: 1411.3727] [INSPIRE].

[48] H.M. Lee and M.-S. Seo, Communication with SIMP dark mesons via $Z^{\prime}$-portal, Phys. Lett. B 748 (2015) 316 [arXiv: 1504.00745] [InSPIRE].

[49] Y. Hochberg, E. Kuflik and H. Murayama, SIMP Spectroscopy, JHEP 05 (2016) 090 [arXiv: 1512.07917] [INSPIRE].

[50] S.-M. Choi and H.M. Lee, SIMP dark matter with gauged $Z_{3}$ symmetry, JHEP 09 (2015) 063 [arXiv: 1505.00960] [INSPIRE].

[51] S.-M. Choi, H.M. Lee and M.-S. Seo, Cosmic abundances of SIMP dark matter, JHEP 04 (2017) 154 [arXiv: 1702.07860] [InSPIRE].

[52] R.T. D'Agnolo and J.T. Ruderman, Light Dark Matter from Forbidden Channels, Phys. Rev. Lett. 115 (2015) 061301 [arXiv: 1505.07107] [INSPIRE].

[53] S.-M. Choi, Y.-J. Kang and H.M. Lee, On thermal production of self-interacting dark matter, JHEP 12 (2016) 099 [arXiv: 1610.04748] [INSPIRE].

[54] N. Bernal, C. Garcia-Cely and R. Rosenfeld, WIMP and SIMP Dark Matter from the Spontaneous Breaking of a Global Group, JCAP 04 (2015) 012 [arXiv:1501.01973] [INSPIRE].

[55] N. Bernal, X. Chu, C. Garcia-Cely, T. Hambye and B. Zaldivar, Production Regimes for Self-Interacting Dark Matter, JCAP 03 (2016) 018 [arXiv:1510.08063] [INSPIRE].

[56] S.-M. Choi and H.M. Lee, Resonant SIMP dark matter, Phys. Lett. B 758 (2016) 47 [arXiv: 1601.03566] [INSPIRE].

[57] A. Berlin, N. Blinov, S. Gori, P. Schuster and N. Toro, Cosmology and Accelerator Tests of Strongly Interacting Dark Matter, Phys. Rev. D 97 (2018) 055033 [arXiv:1801.05805] [INSPIRE].

[58] S.-M. Choi, H.M. Lee, P. Ko and A. Natale, Resolving phenomenological problems with strongly-interacting-massive-particle models with dark vector resonances, Phys. Rev. D 98 (2018) 015034 [arXiv: 1801.07726] [INSPIRE].

[59] Y. Hochberg, E. Kuflik and H. Murayama, Twin Higgs model with strongly interacting massive particle dark matter, Phys. Rev. D 99 (2019) 015005 [arXiv:1805.09345] [INSPIRE].

[60] Y. Hochberg, E. Kuflik, R. Mcgehee, H. Murayama and K. Schutz, Strongly interacting massive particles through the axion portal, Phys. Rev. D 98 (2018) 115031 [arXiv: 1806.10139] [INSPIRE].

[61] S.-M. Choi et al., Vector SIMP dark matter, JHEP 10 (2017) 162 [arXiv:1707.01434] [INSPIRE].

[62] D.N. Spergel and P.J. Steinhardt, Observational evidence for selfinteracting cold dark matter, Phys. Rev. Lett. 84 (2000) 3760 [astro-ph/9909386] [INSPIRE].

[63] W.J.G. de Blok, The Core-Cusp Problem, Adv. Astron. 2010 (2010) 789293 [arXiv: 0910.3538] [INSPIRE].

[64] M. Boylan-Kolchin, J.S. Bullock and M. Kaplinghat, Too big to fail? The puzzling darkness of massive Milky Way subhaloes, Mon. Not. Roy. Astron. Soc. 415 (2011) L40 [arXiv:1103.0007] [INSPIRE]. 
[65] D.H. Weinberg, J.S. Bullock, F. Governato, R. Kuzio de Naray and A.H.G. Peter, Cold dark matter: controversies on small scales, Proc. Nat. Acad. Sci. 112 (2015) 12249 [arXiv: 1306.0913] [INSPIRE].

[66] M. Rocha et al., Cosmological Simulations with Self-Interacting Dark Matter I: Constant Density Cores and Substructure, Mon. Not. Roy. Astron. Soc. 430 (2013) 81 [arXiv: 1208.3025] [INSPIRE].

[67] E. Del Nobile, M. Kaplinghat and H.-B. Yu, Direct Detection Signatures of Self-Interacting Dark Matter with a Light Mediator, JCAP 10 (2015) 055 [arXiv:1507.04007] [INSPIRE].

[68] S. Tulin and H.-B. Yu, Dark Matter Self-interactions and Small Scale Structure, Phys. Rept. 730 (2018) 1 [arXiv: 1705. 02358] [inSPIRE].

[69] F. Elahi and S. Khatibi, Multi-Component Dark Matter in a Non-Abelian Dark Sector, arXiv: 1902.04384 [INSPIRE].

[70] P. Ko and Y. Tang, Residual Non-Abelian Dark Matter and Dark Radiation, Phys. Lett. B 768 (2017) 12 [arXiv:1609.02307] [INSPIRE].

[71] R. Massey et al., The behaviour of dark matter associated with four bright cluster galaxies in the $10 \mathrm{kpc}$ core of Abell 382\%, Mon. Not. Roy. Astron. Soc. 449 (2015) 3393 [arXiv: 1504.03388] [INSPIRE].

[72] F. Kahlhoefer, K. Schmidt-Hoberg, J. Kummer and S. Sarkar, On the interpretation of dark matter self-interactions in Abell 3827, Mon. Not. Roy. Astron. Soc. 452 (2015) L54 [arXiv: 1504.06576] [INSPIRE].

[73] R. Massey et al., Dark matter dynamics in Abell 3827: new data consistent with standard cold dark matter, Mon. Not. Roy. Astron. Soc. 477 (2018) 669 [arXiv:1708. 04245] [INSPIRE].

[74] M. Markevitch et al., Direct constraints on the dark matter self-interaction cross-section from the merging galaxy cluster 1E065\%-56, Astrophys. J. 606 (2004) 819 [astro-ph/0309303] [INSPIRE].

[75] D. Clowe, A. Gonzalez and M. Markevitch, Weak lensing mass reconstruction of the interacting cluster 1E0657-558: Direct evidence for the existence of dark matter, Astrophys. J. 604 (2004) 596 [astro-ph/0312273] [INSPIRE].

[76] S.W. Randall, M. Markevitch, D. Clowe, A.H. Gonzalez and M. Bradac, Constraints on the Self-Interaction Cross-Section of Dark Matter from Numerical Simulations of the Merging Galaxy Cluster 1E 0657-56, Astrophys. J. 679 (2008) 1173 [arXiv:0704.0261] [InSPIRE].

[77] A.H.G. Peter, M. Rocha, J.S. Bullock and M. Kaplinghat, Cosmological Simulations with Self-Interacting Dark Matter II: Halo Shapes vs. Observations, Mon. Not. Roy. Astron. Soc. 430 (2013) 105 [arXiv:1208.3026] [INSPIRE].

[78] Particle Data Group collaboration, Review of Particle Physics, Phys. Rev. D 98 (2018) 030001 [INSPIRE].

[79] BaBAR collaboration, Search for Invisible Decays of a Dark Photon Produced in $e^{+} e^{-}$ Collisions at BaBar, Phys. Rev. Lett. 119 (2017) 131804 [arXiv: 1702.03327] [INSPIRE].

[80] BeLLe-II collaboration, The Belle II Physics Book, arXiv:1808.10567 [INSPIRE].

[81] R. Essig, J. Mardon, M. Papucci, T. Volansky and Y.-M. Zhong, Constraining Light Dark Matter with Low-Energy $e^{+} e^{-}$Colliders, JHEP 11 (2013) 167 [arXiv:1309.5084] [INSPIRE]. 
[82] BaBAr collaboration, Search for a Dark Photon in $e^{+} e^{-}$Collisions at BaBar, Phys. Rev. Lett. 113 (2014) 201801 [arXiv: 1406 . 2980] [INSPIRE].

[83] XENON10 collaboration, A search for light dark matter in XENON10 data, Phys. Rev. Lett. 107 (2011) 051301 [Erratum ibid. 110 (2013) 249901] [arXiv:1104.3088] [INSPIRE].

[84] R. Essig, A. Manalaysay, J. Mardon, P. Sorensen and T. Volansky, First Direct Detection Limits on sub-GeV Dark Matter from XENON10, Phys. Rev. Lett. 109 (2012) 021301 [arXiv: 1206.2644] [INSPIRE].

[85] R. Essig, T. Volansky and T.-T. Yu, New Constraints and Prospects for sub-GeV Dark Matter Scattering off Electrons in Xenon, Phys. Rev. D 96 (2017) 043017 [arXiv: 1703.00910] [INSPIRE].

[86] T.-T. Yu, Direct Detection of sub-GeV Dark Matter, talk at Light Dark World International Forum 2018, KAIST, 17-21 December 2018.

[87] SENSEI collaboration, SENSEI: First Direct-Detection Constraints on sub-GeV Dark Matter from a Surface Run, Phys. Rev. Lett. 121 (2018) 061803 [arXiv:1804.00088] [INSPIRE].

[88] H.E. Logan, TASI 2013 lectures on Higgs physics within and beyond the Standard Model, arXiv:1406.1786 [INSPIRE]. 